\title{
Hamiltonian surface charges using external sources.
}

\author{
Cédric Troessaert $^{a}$ \\ Centro de Estudios Científicos (CECs) \\ Arturo Prat 514, Valdivia, Chile \\ troessaert@cecs.cl
}

\begin{abstract}
In this work, we interpret part of the boundary conditions as external sources in order to solve the integrability problem present in the computation of surface charges associated to gauge symmetries in the hamiltonian formalism. We start by describing the hamiltonian structure of external symmetries preserving the action up to a transformation of the external sources of the theory. We then extend these results to the computation of surface charges for field theories with non-trivial boundary conditions.
\end{abstract}




\section{Contents}

1 Introduction 3

2 Hamiltonian theory with external sources 4

3 Boundary conditions as external sources 10

3.1 Generalized differentiable functionals . . . . . . . . . . . . . 10

3.2 Example: scalar field . . . . . . . . . . . . . . . . 17

4 Surface charges for gauge theories 19

4.1 Symmetries of gauge theories . . . . . . . . . . . . . . . 19

4.2 Gauge field theories . . . . . . . . . . . . . . . . 22

4.3 Link with the non-integrability of surface charge . . . . . . . . . 28

Acknowledgments

A Algebra for the poisson bracket with sources 29

B Variation of the modified brackets 29

C Jacobi identity of the modified bracket

D Jacobi identity for gauge field theories 32 


\section{Introduction}

In field theories, associating conserved generators to gauge symmetries is a long standing problem. The main issue is that the bulk part of the generator will be proportional to the constraints of the theory: evaluated on solutions the associated conserved quantity will be zero. At first sight, this does not seem so bad. However, it means that, for instance, the notion of electric charge in Maxwell's theory or energy in Einstein's theory both disappear.

In hamiltonian formalism, a partial solution to the problem has been developped in [1] and [2]. The idea is that generators must be supplemented with a boundary term in order to be differentiable. The on-shell value of this boundary term is then the associated conserved quantity. Applying this to Einstein's theory in 4D, one recovers the ADM value for the mass [1, 3].

When applying this idea to compute conserved quantities, one has to select a suitable set of boundary conditions. This choice is the key factor: if the boundary conditions are too restrictive, this will reduce the set of available symmetries; if the boundary conditions are too lax, the differentiability condition on the generators will be too strong and the set of symmetries for which we can associate conserved quantities will be small. Looking for a good set of boundary conditions is searching an optimum point of these two tendencies. This problem is often refered as the integrability problem of surface charges as the selection of the boundary term to form a differentiable generator involves solving an integrability equation on the space of field configurations. The references [4, 5] contain a few examples of integrability problems in the hamiltonian formalism.

In this work, we want to present a different approach. The first part of the idea is to treat boundary conditions as external sources. This is certainly reasonable and has already been used, for instance in AdS/CFT (see [6] and subsequent literature). The second part is to allow symmetries to act on the sources. The obtained symmetries are not really symmetries of the theory and will no give rise to conserved quantities. They are symmetries that send a solution of the equations of motion for one value of the sources to a solution with a different value of the sources. They are symmetries between different theories and, in this work, we will call them external symmetries. We will see that they are generated by canonical generators and that the algebra of these generators forms a possibly extended representation of the algebra of the external symmetries.

Treating boundary conditions as external sources introduces two layers of boundary conditions. The external layer describes all field configurations for all possible values of the boundary conditions (Dirichlet, ...). This is the set of conditions that must be preserved by the external symmetries. The internal layer describes the field configurations for each theory: for each specific value of the boundary conditions. This is the set of boundary 
conditions satisfied by the dynamical part of the fields and is related to the differentiability condition of the generators. In other words, we decoupled the two effects of the boundary conditions in the integrability problem and introduced a lot more possibilities.

The first part of this work contains a definition and study of the notion of external symmetries in hamiltonian theories with a finite number of degrees of freedom. We show that, under reasonable assumptions, these symmetries are generated by canonical generators. We also introduce a modified poisson bracket to compute the algebra of these associated generators. This algebra forms a possibly extended representation of the algebra of the external symmetries.

In the second part, we study hamiltonian field theories without gauge freedom. We promote boundary conditions to external sources and use the results we obtained earlier to define external symmetries in this case. As before, we introduce a modified poisson bracket and compute the algebra of the generators. We then provide an example by applying the results to a scalar field theory.

The last part contains the study of gauge theories. Due to possible interactions between dynamical fields and lagrange multipliers through boundary conditions, we have to keep the lagrange multipliers explicitly in our analysis. To this end, we start with a small generalisation of standard results concerning conserved quantities in gauge theories with a finite number of degrees of freedom. We then combine this with the notion of external symmetries developed in the previous sections. As before, we compute the algebra of the generators of external symmetries and show that it forms a possibly extended representation of the algebra of the associated symmetries.

\section{Hamiltonian theory with external sources}

In this section, we will describe the hamiltonian theory in presence of external sources. We will focus on mechanical systems with a finite number of degrees of freedom.

A general system has the following action:

$$
S\left[q^{i}, p_{i} ; j^{\alpha}\right]=\int d t\left\{\dot{q}^{i} p_{i}-H\left(t, q^{i}, p_{i} ; j^{\alpha}, d_{t} j^{\alpha}, \ldots, d_{t}^{k} j^{\alpha}\right)\right\},
$$

where $j^{\alpha}$ is an external source: it is not varied when deriving the equations of motions. If we only work with transformations that preserve exactly the form of the action, sources included, the usual hamiltonian theory of charges is well behaved. We are able to associate conserved canonical generators and compute their algebra for each fixed value of $j$. The general picture is a symmetry group for $j=0$ that, when $j \neq 0$, is broken to the subgroup preserving this particular value of $j$. 
There exists systems where we can keep all the symmetries if we allow the transformations to act on the sources. For instance electromagnetism in presence of an external current

$$
S\left[A_{\mu} ; j^{\nu}\right]=\int d^{n} x\left(-\frac{1}{4} F_{\mu \nu} F^{\mu \nu}+A_{\mu} j^{\mu}\right),
$$

is invariant under the full Poincaré algebra if we allow the transformations to act on $j^{\mu}$. However, these transformations don't really preserve the action, they send one value of the sources to a different one: they are symmetries between different problems. Our goal in this section is to see how we can extend the hamiltonian theory of charges to these generalized symmetries.

An external symmetry $\delta_{G}$ of the action (2.1) will be defined as a transformation of the form

$$
\begin{aligned}
\delta_{G} q^{i} & =Q^{i}\left(t, q, d_{t} q, \ldots, p, d_{t} p, \ldots, j, d_{t} j, \ldots\right), \\
\delta_{G} p_{i} & =P_{i}\left(t, q, d_{t} q, \ldots, p, d_{t} p, \ldots, j, d_{t} j, \ldots\right), \\
\delta_{G} j^{\alpha} & =J^{\alpha}\left(t, q, d_{t} q, \ldots, p, d_{t} p, \ldots, j, d_{t} j, \ldots\right),
\end{aligned}
$$

that preserves the integrand of the action up to a time derivative or a function of the sources

$$
\delta_{G}\left\{\dot{q}^{i} p_{i}-H\left(t, q^{i}, p_{i} ; j^{\alpha}, d_{t} j^{\alpha}, \ldots, d_{t}^{k} j^{\alpha}\right)\right\}=\frac{d}{d t} U(t, q, p ; j, \ldots)+V_{G}\left(j, d_{t} j, \ldots\right) .
$$

The most important property in the usual case is that a symmetry of the action is a symmetry of the equations of motion. This is coming from the identity

$$
\delta_{G} \frac{\delta}{\delta z^{A}} L=\frac{\delta}{\delta z^{A}} \delta_{G} L+\sum_{k=0}^{\infty}\left(-d_{t}\right)^{k}\left(\frac{\partial Z^{B}}{\partial\left(d_{t}\right)^{k} z^{A}} \frac{\delta L}{\delta z^{B}}\right)
$$

where $z^{A}=\left(q^{i}, p_{i}\right)$ and $Z^{A}=\left(Q^{i}, P_{i}\right)$. If $\delta_{G} L=d_{t} U$, this identity reduces to

$$
\delta_{G} \frac{\delta}{\delta z^{A}} L=\sum_{k=0}^{\infty}\left(-d_{t}\right)^{k}\left(\frac{\partial Z^{B}}{\partial\left(d_{t}\right)^{k} z^{A}} \frac{\delta L}{\delta z^{B}}\right)
$$

which is zero on the $\mathrm{EOM} \frac{\delta L}{\delta z^{A}} \approx 0$. In our case, the identity (2.7) becomes

$$
\delta \frac{\delta}{\delta z^{A}} L=\sum_{k=0}^{\infty}\left(-d_{t}\right)^{k}\left(\frac{\partial Z^{B}}{\partial\left(d_{t}\right)^{k} z^{A}} \frac{\delta L}{\delta z^{B}}\right)+\sum_{k=0}^{\infty}\left(-d_{t}\right)^{k}\left(\frac{\partial J^{\alpha}}{\partial\left(d_{t}\right)^{k} z^{A}} \frac{\delta L}{\delta j^{\alpha}}\right) .
$$

This is zero on the equations of motion if $J^{\alpha}$ is independent of the dynamical fields. The sources have to transform without involving the dynamical fields.

This is a natural restriction to impose on the transformations. In this case, one problem (the variational problem for one value of $j^{\alpha}$ ) will be send by the transformation to another problem (same variational problem for a different value of $j^{\alpha}$ ). If the transformation of 
$j^{\alpha}$ is allowed to depend on the dynamical fields, all the possible values of the dynamical fields for one value of $j^{\alpha}$ will be send to different values of the sources, to different problems. This will completely destroy our variational theory. In the following, we will only work with transformations where $J^{\alpha}$ is independent of $z^{A}$. Another useful way to encode this information is:

$$
\left[\delta, \delta_{G}\right] j^{\alpha}=0 \quad \forall \delta \quad \text { s.t. } \quad \delta j^{\alpha}=0 .
$$

For any action, we have trivial symmetry transformations

$$
\delta_{M} z^{A}=M^{A B}\left(\frac{\delta L}{\delta z^{B}}\right) \quad \delta_{M} j^{\alpha}=0,
$$

where the operator $M^{A B}$ is an anti-self-adjoint operator:

$$
X_{A} M^{A B}\left(Y_{B}\right)=-Y_{A} M^{A B}\left(X_{B}\right)+\frac{d}{d t} \Xi_{M}(X, Y) .
$$

They can be used to remove all dependence of $Z^{A}$ in the time derivatives of the fields $z^{A}$. Because of this, the most general transformation we will consider is of the form

$$
\delta_{G} z^{A}=Z^{A}\left(t, z^{B}, j^{\alpha}, d_{t} j^{\alpha}, \ldots,\left(d_{t}\right)^{l} j^{\alpha}\right), \quad \delta_{G} j^{\alpha}=J^{\alpha}\left(t, j^{\beta}, d_{t} j^{\beta}, \ldots,\left(d_{t}\right)^{l} j^{\beta}\right) .
$$

Equation (2.6), can be rewritten as

$$
\dot{Q}^{i} p_{i}+\dot{q}^{i} P_{i}-\frac{\partial H}{\partial q^{i}} Q^{i}-\frac{\partial H}{\partial p_{i}} P_{i}-\delta_{G}^{j} H=\frac{\partial U}{\partial q^{i}} \dot{q}^{i}+\frac{\partial U}{\partial p_{i}} \dot{p}_{i}+\frac{\partial U}{\partial t}+\delta_{t}^{j} U+V_{G},
$$

where we have introduced the following notations

$$
\begin{aligned}
\delta_{G}^{j} & =J^{\alpha} \frac{\partial}{\partial j^{\alpha}}+d_{t} J^{\alpha} \frac{\partial}{\partial d_{t} j^{\alpha}}+d_{t}^{2} J^{\alpha} \frac{\partial}{\partial d_{t}^{2} j^{\alpha}}+\ldots \\
\delta_{t}^{j} & =d_{t} j^{\alpha} \frac{\partial}{\partial j^{\alpha}}+d_{t}^{2} j^{\alpha} \frac{\partial}{\partial d_{t} j^{\alpha}}+d_{t}^{3} j^{\alpha} \frac{\partial}{\partial d_{t}^{2} j^{\alpha}}+\ldots
\end{aligned}
$$

This equation is valid for all values of $q^{i}, p_{i}, \dot{q}^{i}$ and $\dot{p}_{i}$. It can be decomposed into the following equations:

$$
\begin{aligned}
\frac{\partial Q^{j}}{\partial q^{i}} p_{j}+P_{i} & =\frac{\partial U}{\partial q^{i}}, \\
\frac{\partial Q^{j}}{\partial p_{i}} p_{j} & =\frac{\partial U}{\partial p_{i}}, \\
\partial_{t} Q^{i} p_{i}+\delta_{t}^{j} Q^{i} p_{i}-\frac{\partial H}{\partial q^{i}} Q^{i}-\frac{\partial H}{\partial p_{i}} P_{i}-\delta_{G}^{j} H & =\frac{\partial U}{\partial t}+\delta_{t}^{j} U+V_{G} .
\end{aligned}
$$

The first two equations imply that the transformation of the hamiltonian variables $\left(q^{i}, p_{i}\right)$ is symplectic and has as generator $G=Q^{j} p_{j}-U$. Using this on the last equation leads to

$$
\partial_{t} G+\{G, H\}+\delta_{t}^{j} G-\delta_{G}^{j} H=V_{G},
$$


where we have introduced the usual poisson bracket given by:

$$
\{F, G\}=\frac{\partial F}{\partial q^{i}} \frac{\partial G}{\partial p_{i}}-\frac{\partial G}{\partial q^{i}} \frac{\partial F}{\partial p_{i}}=\frac{\partial F}{\partial z^{A}} \sigma^{A B} \frac{\partial G}{\partial z^{B}},
$$

with $\sigma^{A B}$ the poisson structure.

We have the following result:

Theorem 2.1. A transformation of the form

$$
\delta_{G} z^{A}=Z^{A}\left(t, z, j, \partial_{t} j, \ldots, \partial_{t}^{l} j\right) \quad \text { and } \quad \delta_{G} j^{\alpha}=J^{\alpha}\left(t, j, \partial_{t} j, \ldots, \partial_{t}^{l} j\right),
$$

preserves the action in the sense (2.6) if and only if there exists a generator $G$ such that it is the hamiltonian generator of the transformation of the canonical variables and it satisfies (2.17), (2.18) and (2.20).

Such transformations will be called external symmetries. Equation (2.20) is the equivalent of the conservation of $G$ in the absence of external sources. It can be rewritten as:

$$
\delta_{t} G[z ; j]=\delta_{G}^{j} H[z ; j]+V_{G}[j], \quad \delta_{t} \equiv \frac{\partial}{\partial t}+\sigma^{A B} \frac{\partial H}{\partial z^{B}} \frac{\partial}{\partial z^{A}}+\delta_{t}^{j} .
$$

Giving the generator $G$ is not enough to describe the transformation, one must also supply the variation of the sources $J^{\alpha}$ or equivalently the operator $\delta_{G}^{j}$. In the following, when we are referring to a transformation generated by $G$, we will assume that both $G$ and $\delta_{G}^{j}$ are known. The generator $G$ is defined up to a function of the sources: both $G$ and $G+K[j]$ generate the same transformation.

Let's compute the generator associated to the commutator of two transformations $\left(F, \delta_{F}^{j}\right)$ and $\left(G, \delta_{G}^{j}\right)$ :

$$
\begin{aligned}
\delta_{[F, G]} z^{A} & =\delta_{F}\left\{z^{A}, G[z ; j]\right\}-F \leftrightarrow G \\
& =\left\{\left\{z^{A}, F[z ; j]\right\}, G[z ; j]\right\}+\left\{z^{A},\{G[z ; j], F[z ; j]\}+\delta_{F}^{j} G[z ; j]\right\}-F \leftrightarrow G \\
& =\left\{z^{A},\{G[z ; j], F[z ; j]\}+\delta_{F}^{j} G[z ; j]-\delta_{G}^{j} F[z ; j]\right\} .
\end{aligned}
$$

The canonical generator of $\delta_{[F, G]} z^{A}$ is given by:

$$
\{G[z ; j], F[z ; j]\}+\delta_{F}^{j} G[z ; j]-\delta_{G}^{j} F[z ; j] .
$$

Following this, we introduce a new poisson Bracket for the generators in the presence of external sources:

$$
\{F, G\}_{j} \equiv\{F, G\}+\delta_{G}^{j} F-\delta_{F}^{j} G
$$


As we said earlier, the generators are associated with specific transformations of the sources, the bracket of the generator only is not well-defined. It should be extended to couples $\left(G, \delta_{G}^{j}\right)$. The couple generating the transformation $\delta_{[F, G]}$ is given by:

$$
\left[\left(F, \delta_{F}^{j}\right),\left(G, \delta_{G}^{j}\right)\right]=\left(\{G, F\}_{j},\left[\delta_{F}^{j}, \delta_{G}^{j}\right]\right) .
$$

This bracket is obviously antisymmetric and it can be checked that it satisfies the Jacobi identity. If we identify the hamiltonian $H$ with the generator of time translations, we must supply it with the right variation of the source namely $\delta_{H} j^{\alpha} \equiv d_{t} j^{\alpha}$, which implies $\delta_{H}^{j}=\delta_{t}^{j}$. Using our new poisson Bracket, equation (2.20) becomes

$$
\partial_{t} G+\{G, H\}_{j}=V_{G}[j] .
$$

In the usual case, when considering symmetries of the action preserving the sources $\delta_{G}^{j}=0$, we also have the possibility to add this extra term $V_{G}$. In order to preserve the EOM, we need $V_{G}$ to be a constant. The analysis done above goes through and we obtain this constant on the right hand side of the conservation equation (2.28) with the unmodified poisson bracket. In this case, the constant can be absorbed in the generator by adding to it a term of the form $-t V_{G}$ allowing us to recover the usual result. For the more general case considered in this work, due to the presence of the sources and their arbitrary time dependence, we cannot get rid of this extra term.

The set of external symmetries (2.13) form an algebra $\mathcal{G}$ of symmetries of the set of theories parametrized by $j^{\alpha}$. To any of these transformations, we can associate a couple $\left(G, \delta_{G}^{j}\right)$. These couples associated to the commutator

$$
\left[\left(F, \delta_{F}^{j}\right),\left(G, \delta_{G}^{j}\right)\right]=\left(\{G, F\}_{j},\left[\delta_{F}^{j}, \delta_{G}^{j}\right]\right)
$$

form a representation of $\mathcal{G}$ (The only property missing is that $\left(\{G, F\}_{j},\left[\delta_{F}^{j}, \delta_{G}^{j}\right]\right)$ satisfies (2.28) which is proven in appendix A). This representation has room for extensions. This is coming from the fact that we can add any function of the sources to a generator $G$.

Theorem 2.2. If $G_{i}$ forms a generating set of the algebra $\mathcal{G}$, we have in general

$$
\left(\left\{G_{2}, G_{1}\right\}_{j},\left[\delta_{1}^{j}, \delta_{2}^{j}\right]\right)=\left(G_{[1,2]}+K_{1,2}[j], \delta_{[1,2]}^{j}\right),
$$

with $K_{1,2}$ antisymmetric and

$$
K_{[1,2], 3}+\delta_{3}^{j} K_{1,2}+\text { cyclic }=0 .
$$

The cyclic identity of the extension $K_{1,2}$ comes from the Jacobi identity of the modified bracket. This is an abelian extension based on the representation of $\mathcal{G}$ on the sources $j$ [7]. 
As an application, let's consider a point particle on which we act with an external force $f_{i}(t)$ :

$$
S\left[q^{i}, p_{i} ; f_{i}\right]=\int d t\left(\dot{q}^{i} p_{i}-\frac{p_{i} p^{i}}{2 m}+q^{i} f_{i}\right)
$$

where indices are raised and lowered with the Kronecker delta. The equations of motion are

$$
\begin{aligned}
& \dot{q}^{i}=\frac{p^{i}}{m}, \\
& \dot{p}_{i}=f_{i},
\end{aligned}
$$

and, if we remove $p$, we obtain the famous Newton equations

$$
m d_{t}^{2} q^{i}=f^{i}
$$

The presence of this source term in the action spoils galilean symmetries but using the above results, we can still associate generators to them. The galilean transformations are:

$$
\begin{aligned}
\delta_{a, v, \omega} q^{i} & =a^{i}+t v^{i}-\omega_{j}^{i} q^{j}, \\
\delta_{a, v, \omega} p_{i} & =m v_{i}+\omega_{i}^{j} p_{j}, \\
\delta_{a, v, \omega} f_{i} & =\omega_{i}^{j} f_{j},
\end{aligned}
$$

where $a^{i}, v^{i}$ and $\omega_{i j}$ with $\omega_{(i j)}=0$ respectively parametrize translations, boosts and rotations. These transformations are of the form (2.13) and preserve the action in the sense (2.6):

$$
\delta_{a, v, \omega} L=\frac{d}{d t}\left(m q^{i} v_{i}\right)+\left(a^{i}+t v^{i}\right) f_{i}
$$

We can then associate non-conserved generators

$$
\begin{aligned}
G_{a, v, \omega} & =\left(a^{i}-\omega^{i}{ }_{j} q^{j}\right) p_{i}+t v^{i} p_{i}-m v_{i} q^{i}, \\
d_{t} G_{a, v, \omega} & =\left(a^{i}+t v^{i}-\omega_{j}^{i} q^{j}\right) f_{i} .
\end{aligned}
$$

Part of the evolution of $G_{a, v, \omega}$ is just a rewriting of the well-known results concerning the evolution of the momentum and angular momentum of a point particle in presence of an external force. In this case, the new poisson bracket of the generators is the same than the old one and the algebra closes with a central extension between the boosts and the translations [7]:

$$
\left\{G_{1}, G_{2}\right\}_{j}=\left(\widehat{a}^{i}-\widehat{\omega}_{j}^{i} q^{j}\right) p_{i}+t \widehat{v}^{i} p_{i}-m \widehat{v}_{i} q^{i}+m\left(a_{1}^{i} v_{2 i}-a_{2}^{i} v_{1 i}\right),
$$

where

$$
\begin{gathered}
\widehat{a}^{i}=\omega_{2 j}^{i} a_{1}^{j}-\omega_{1 j}^{i} a_{2}^{j}, \quad \widehat{v}^{i}=\omega_{2 j}^{i} v_{1}^{j}-\omega_{1 j}^{i} v_{2}^{j}, \\
\widehat{\omega}^{i}{ }_{j}=\omega_{2 k}^{i} \omega_{1 j}^{k}-\omega_{1 k}^{i} \omega_{2 j}^{k} .
\end{gathered}
$$




\section{Boundary conditions as external sources}

In this section, we will use the previous results to generalize the hamiltonian theory of charges to symmetries not preserving boundary conditions. The first subsection introduces the theoretical objects needed and gives the results concerning external symmetries when boundary conditions are treated as external sources. The second subsection contains an application: we compute the external symmetries for a scalar field contained in a sphere with Dirichlet boundary conditions.

From here on, we will use the conventions introduced in appendix A of $[8]$ in order to describe spatial field configurations.

\subsection{Generalized differentiable functionals}

We will consider hamiltonian systems defined on a manifold $\Sigma$ with boundaries $\partial \Sigma$ at a finite distance. Functionals of the canonical fields $z^{A}(x)$ are assumed to have the usual poisson bracket

$$
\{F, G\}=\int_{\Sigma} d^{n} x \frac{\delta F}{\delta z^{A}} \sigma^{A B} \frac{\delta G}{\delta z^{B}},
$$

with $\sigma^{A B}$ a constant, anti-symmetric and invertible matrix. We will also assume a set of boundary conditions on $z^{A}$. The results we will describe in this section can be extended easily to boundaries at infinity and asymptotic conditions.

The Hamiltonian theory of charges for field theories introduced in [1] and further developed in [2] is based on the idea that only differentiable generators are allowed in the poisson bracket. A differentiable generator $G[z]$ is a functional such that:

- the boundary term produced in its variation is zero:

$$
\delta G=\int_{\Sigma} d^{n} x \frac{\delta G}{\delta z^{A}} \delta z^{A},
$$

where $\delta z^{A}$ respects the boundary conditions imposed on $z^{A}$.

- its associated hamiltonian vector field $\delta_{G}$ with

$$
\delta_{G}=\partial_{(i)} G^{A} \frac{\partial^{S}}{\partial z_{(i)}^{A}}, \quad G^{A}=\sigma^{A B} \frac{\delta G}{\delta z^{B}},
$$

preserves the boundary conditions on $z^{A}$.

With this definition, the hamiltonian theory is well behaved. However, there are cases where the above conditions are too restrictive. In the following, we will relax the second condition by treating part of the boundary conditions on $z^{A}$ as external sources. 
We will consider boundary conditions on the bulk fields $z^{A}$ parametrized by a set of non dynamical boundary fields $\zeta^{\alpha}$ :

$$
\left.\chi_{I}^{\alpha}(z)\right|_{\partial \Sigma}=\zeta^{\alpha}
$$

We will assume the operators $\chi_{I}^{\alpha}$ to be local and independent of time. The standard cases are Dirichlet or Newmann boundary conditions. In order to guarantee smooth solutions, we have to supplement these "internal" boundary conditions with extra "external" boundary conditions:

$$
\begin{gathered}
\chi_{E}^{k, \alpha}=\left(d_{t}-\delta_{t}\right)^{k} \chi_{I}^{\alpha} \quad \forall k>0, \quad \delta_{t}=\frac{\partial}{\partial t}+\delta_{H}, \\
\left.\chi_{E}^{k, \alpha}\right|_{\partial \Sigma}=0 \quad \forall k>\left.0 \quad \Leftrightarrow \quad\left(d_{t}^{k}-\delta_{t}^{l}\right) \chi_{I}^{\alpha}\right|_{\partial \Sigma}=0 \quad \forall k>0,
\end{gathered}
$$

where $d_{t}$ is the total time derivative and $\delta_{H}$ is the variation generated by the hamiltonian. Once a value of the boundary field $\zeta^{\alpha}$ has been chosen, these extra boundary conditions become:

$$
\left.\delta_{t}^{k} \chi_{I}^{\alpha}\right|_{\partial \Sigma}=d_{t}^{k} \zeta^{\alpha} \quad \forall k>0
$$

which usually are boundary conditions on normal derivatives of $z^{A}$. However, if $\zeta^{\alpha}$ is allowed to vary, they don't impose any restriction on configurations $z^{A}(k)$ at fixed time. When it doesn't lead to confusion, we will use $\chi_{E}$ to denote the set of external boundary conditions $\chi_{E}^{k, \alpha}$.

In the following, we will call variations of the fields $\delta z^{A}$ preserving the internal boundary conditions the variations satisfying:

$$
\left.\delta \chi_{I}^{\alpha}\right|_{\partial \Sigma}=0,\left.\quad \delta \chi_{E}\right|_{\partial \Sigma}=0, \quad \forall z \text { s.t. }\left.\chi_{E}\right|_{\partial \Sigma}=0 .
$$

In general, if we restrict ourselves to configurations associated to a fixed value of $\zeta^{\alpha}$, the set of variations obtained is bigger than the one defined in (3.8). In the following, we will only consider operators $\chi_{I}^{\alpha}$ such that these two sets are equal.

Inspired by the results of the previous section, we extend the definition of differentiable generator to:

Definition 3.1. A generalized differentiable generator $G[z]$ is a functional such that, for all variation $\delta z^{A}$ preserving the internal boundary conditions, we have

$$
\delta G=\int_{\Sigma} d^{n} x \frac{\delta G}{\delta z^{A}} \delta z^{A}, \quad \forall z \text { s.t. }\left.\chi_{E}\right|_{\partial \Sigma}=0
$$

and

$$
\left.\delta_{G} \chi_{E}\right|_{\partial \Sigma}=0,\left.\quad\left[\delta, \delta_{G}\right] \chi_{I}^{\alpha}(z)\right|_{\partial \Sigma}=0, \quad \forall z \text { s.t. }\left.\chi_{E}\right|_{\partial \Sigma}=0,
$$

where $\delta_{G} z^{A} \equiv G^{A}=\sigma^{A B} \frac{\delta G}{\delta z^{B}}$ is the transformation generated by $G$. 
The first condition (3.9) guarantees that the variational principle generating the transformation $\delta_{G} z^{A}$ is well-defined for each value of the boundary conditions $\zeta^{\alpha}$ :

$$
S_{G}\left[z^{A} ; \zeta^{\alpha}\right]=\int d t \int_{\Sigma} d^{n} x\left(z^{A} \sigma_{A B} \partial_{s} z^{B}-G[z]\right)
$$

where $\sigma_{A B}$ is the inverse of $\sigma^{A B}$. The second condition (3.10) is the requirement that the transformation of the sources depends on the sources only: it is the equivalent of equation (2.10) of the previous section. The differentiable generators in the sense of Regge-Teitelboim [1] are the generalized differentiable generators preserving the internal boundary conditions: $\left.\delta_{G} \chi_{I}^{\alpha}(z)\right|_{\partial \Sigma}=0$.

By analogy with the results of the previous section, we want to define the following modified bracket of generalized differentiable functionals $F$ and $G$ :

$$
\{F, G\}_{\zeta}=\{F, G\}+\delta_{G}^{\zeta} F-\delta_{F}^{\zeta} G
$$

where $\delta^{\zeta}$ is the variation of the functionals only hitting the sources $\zeta^{\alpha}$. However, whereas in the previous section the separation between dynamical variables and sources was easily done, in this case, $\zeta^{\alpha}$ and $z^{A}$ are linked by boundary conditions which means that the action of $\delta_{G}^{\zeta}$ is hard to identify.

To this end, let's consider a general variation $\delta z^{A}$ preserving only the external boundary conditions $\left.\delta \chi_{E}\right|_{\partial \Sigma}=0$. Equation (3.9) implies that the boundary term only contains variations of the source $\zeta^{\alpha}$ :

$$
\delta G=\int_{\Sigma} d^{n} x \frac{\delta G}{\delta z^{A}} \delta z^{A}+\oint_{\partial \Sigma}\left(d^{n-1} x\right)_{i} \Theta_{G}^{i}\left[\delta \zeta^{\alpha}\right] .
$$

Let's now consider variations of the form

$$
\delta_{\epsilon} z^{A} \equiv \eta_{\epsilon}(x) \delta z^{A}
$$

where $\eta_{\epsilon}$ are smooth functions that are zero in a neighbourhood of the boundary and such that

$$
\lim _{\epsilon \rightarrow 0} \eta_{\epsilon}(x)=1, \quad \forall x \in \Sigma \backslash \partial \Sigma .
$$

For all values of $\epsilon \neq 0, \delta_{\epsilon} z^{A}$ preserves the internal boundary conditions (3.8) which implies

$$
\lim _{\epsilon \rightarrow 0}\left(\delta-\delta_{\epsilon}\right) G=\oint_{\partial \Sigma}\left(d^{n-1} x\right)_{i} \Theta_{G}^{i}\left[\delta \zeta^{\alpha}\right] .
$$

It means that the source part of the variation of $G[z]$ is only encoded in the boundary term. With this, we define the modified bracket of generalized differentiable functionals $F$ and $G$ as:

$$
\{F, G\}_{\zeta}=\{F, G\}+\oint_{\partial \Sigma}\left(d^{n-1} x\right)_{i} \Theta_{F}^{i}\left[\delta_{G} \zeta^{\alpha}\right]-\oint_{\partial \Sigma}\left(d^{n-1} x\right)_{i} \Theta_{G}^{i}\left[\delta_{F} \zeta^{\alpha}\right]
$$


The boundary terms $\Theta_{F}$ and $\Theta_{G}$ are defined by equation (3.9) for both $F$ and $G$. Because the modification only concerns boundary terms, the variation generated by $\{F, G\}_{\zeta}$ is given by

$$
\delta_{\{F, G\}_{\zeta}}=\left[\delta_{G}, \delta_{F}\right] .
$$

We also see that, for differentiable functionals in the sense of Regge-Teitelboim [1], both variations $\delta_{G} \zeta$ and $\delta_{F} \zeta$ are zero and the bracket (3.17) reduces to the unmodified poisson bracket (3.1).

Theorem 3.2. The modified bracket (3.17) is a well-defined poisson bracket.

Proof. It is manifestly anti-symmetric and it satisfies the Jacobi identity. An easy way to see that is to recognize that this bracket is a particular case of the one introduced in [9] where we reduced the set of available functionals. For completeness, we also give a direct proof in appendix $\mathrm{C}$. The only thing still left to prove is that the modified bracket of two generalized differential functionals is a generalized differentiable functional. The second property (3.10) is easy to check using the Jacobi identity for variations:

$$
\left[\delta_{\{F, G\}_{\zeta}}, \delta\right]=\left[\delta_{G},\left[\delta_{F}, \delta\right]\right]-\left[\delta_{F},\left[\delta_{G}, \delta\right]\right] .
$$

For the first condition (3.9), the analysis is done in appendix B

Now that we have defined our additional structures, we will see how they describe the external symmetries of the system:

$$
S[z]=\int d t\left(\int_{\Sigma} d^{n} x \frac{1}{2} z^{A} \sigma_{A B} \dot{z}^{B}-H[z]\right) .
$$

We will assume that a set of boundary conditions $\chi_{I}^{\alpha}, \chi_{E}$ has been selected such that the action is well-defined under variations preserving the internal boundary conditions. In that case, one can check that the Hamiltonian $H[z]$ is a generalized differentiable functional with $\delta_{H} \zeta^{\alpha}=\dot{\zeta}^{\alpha}$.

In analogy with the previous section, we define:

Definition 3.3. An external symmetry $\delta_{G} z^{A}=Z^{A}(t, z)$ of the action is a transformation such that

$$
\left.\delta_{G} \chi_{E}\right|_{\partial \Sigma}=0,\left.\quad\left[\delta_{G}, \delta\right] \chi_{I}^{\alpha}(z)\right|_{\partial \Sigma}=0,
$$

and

$$
\begin{gathered}
\delta_{G} S[z]=\int d t\left\{\frac{d}{d t} \int_{\Sigma} d^{n} x U(t, z)+\oint_{\partial \Sigma}\left(d^{n-1} x\right)_{i} V_{G}^{i}[z]\right\}, \\
\delta \oint_{\partial \Sigma}\left(d^{n-1} x\right)_{i} V_{G}^{i}(z)=0,
\end{gathered}
$$

for all $\delta$ preserving the internal boundary conditions. 
The last condition (3.23) means that the boundary term $V_{G}$ only depends on the nondynamical boundary fields $\zeta^{\alpha}$. As in the previous section, this is a symmetry between different systems corresponding to different values of the boundary conditions $\zeta^{\alpha}$. $\operatorname{Re}$ mark that compared to the previous section, we don't have to specify the variation of the sources as it is obtained by continuity from the bulk variation.

Theorem 3.4. A transformation of the form (3.21), (3.22) is an external symmetry of the action (3.23) if and only if there exists a generalized differentiable functional $G[z]$ such that:

$$
\begin{gathered}
\delta_{G} z^{A}=\left\{z^{A}, G\right\}=\sigma^{A B} \frac{\delta G}{\delta z^{B}} \\
\partial_{t} G+\{G, H\}_{\zeta}=\oint\left(d^{n-1} x\right)_{i} V_{G}^{i}(z), \quad \delta \oint_{\partial \Sigma}\left(d^{n-1} x\right)_{i} V_{G}^{i}(z)=0
\end{gathered}
$$

where $\partial_{t}$ only hits the explicit dependence in $t$ and $\delta$ span all variations preserving the internal boundary conditions.

Proof. Let's assume that $\delta_{G}$ is an external symmetry of the action, developing the LHS of (3.22), we get

$$
\begin{array}{r}
\int d t\left\{\int_{\Sigma} d^{n} x\left(\frac{1}{2} \sigma_{A B} Z^{A} \dot{z}^{B}+\frac{1}{2} \sigma_{A B} z^{A} d_{t} Z^{B}-Z^{A} \frac{\delta H}{\delta z^{A}}\right)-\oint_{\partial \Sigma}\left(d^{n-1} x\right)_{i} \Theta_{H}^{i}\left[\delta_{G} z\right]\right\} \\
=\int d t\left\{\frac{d}{d t} \int_{\Sigma} d^{n} x U(t, z)+\oint_{\partial \Sigma}\left(d^{n-1} x\right)_{i} V_{G}^{i}(z)\right\} .
\end{array}
$$

Introducing the following functional:

$$
G[z]=\int_{\Sigma} d^{n} x\left\{\frac{1}{2} \sigma_{A B} z^{A} Z^{B}-U(t, z)\right\},
$$

equation (3.26) can be written as

$$
\begin{aligned}
\int d t \frac{d G}{d t}=\int d t\left\{\int _ { \Sigma } d ^ { n } x \left(-\sigma_{A B} Z^{A} \dot{z}^{B}\right.\right. & \left.+Z^{A} \frac{\delta H}{\delta z^{A}}\right) \\
& \left.+\oint_{\partial \Sigma}\left(d^{n-1} x\right)_{i}\left(\Theta_{H}^{i}\left[\delta_{G} z\right]+V_{G}^{i}(z)\right)\right\},
\end{aligned}
$$

where we can expand the LHS to

$$
\int d t \frac{d G}{d t}=\int d t\left\{\frac{\partial G}{\partial t}+\int_{\Sigma} d^{n} x \frac{\delta G}{\delta z^{A}} \dot{z}^{A}+\oint_{\partial \Sigma}\left(d^{n-1} x\right)_{i} \Theta_{G}^{i}[\dot{z}]\right\} .
$$

In the bulk, $\dot{z}^{A}$ is arbitrary, this implies

$$
\frac{\delta G}{\delta z^{B}}=-\sigma_{B A} Z^{A} \quad \Rightarrow \quad Z^{A}=\sigma^{A B} \frac{\delta G}{\delta z^{B}},
$$


which means that $G$ is the canonical generator of the transformation. Putting everything together, the equality (3.26) becomes

$$
\int d t\left[\frac{\partial G}{\partial t}+\{G, H\}_{\zeta}-\oint_{\partial \Sigma}\left(d^{n-1} x\right)_{i} V^{i}(z)\right]=\int d t \oint_{\partial \Sigma}\left(d^{n-1}\right)_{i} \Theta_{G}^{i}\left[\delta_{H} z-\dot{z}\right] .
$$

By construction, the transformation $\delta_{H} z-\dot{z}=\left(\delta_{t}-d_{t}\right) z=\widehat{\delta} z$ preserves all boundary conditions. Moreover, apart from these preservation conditions, it is completely arbitrary: it is an arbitrary variation preserving the internal boundary conditions. As the LHS of (3.31) is independant of $\dot{z}^{A}$, we have

$$
\oint_{\partial \Sigma}\left(d^{n-1}\right)_{i} \Theta_{G}^{i}[\delta z]=0
$$

for all variations $\delta z^{A}$ preserving the internal boundary conditions. The generator $G$ is a generalized differentiable generator. The LHS of (3.31) being zero for all intervals of integration $\left[t_{0}, t_{1}\right]$ leads to:

$$
\partial_{t} G+\{G, H\}_{\zeta}=\oint\left(d^{n-1} x\right)_{i} V_{G}^{i}(z)
$$

which is what we wanted.

For the other direction, let's assume that we have a generalized differentiable generator $G$ satisfying (3.25). The variation of the action under the transformation generated by $G, \delta_{G} z^{A}=G^{A}$, is

$$
\begin{aligned}
& \delta_{G} S=\int d t \int d^{n} x\left\{\frac{1}{2} \sigma_{A B} G^{A} \dot{z}^{B}+\frac{1}{2} \sigma_{A B} z^{A} d_{t} G^{B}-\delta_{G} H\right\} \\
& =\int d t\left\{\frac{d}{d t}\left(\int_{\Sigma} d^{n} x \frac{1}{2} \sigma_{A B} z^{A} G^{B}\right)+\int_{\Sigma} d^{n} x \sigma_{A B} G^{A} \dot{z}^{B}\right. \\
& \left.+\{G, H\}_{\zeta}-\oint_{\partial \Sigma}\left(d^{n-1}\right)_{i} \Theta_{G}^{i}\left[\delta_{H} z\right]\right\} \\
& =\int d t\left\{\frac{d}{d t}\left(\int_{\Sigma} d^{n} x \frac{1}{2} \sigma_{A B} z^{A} G^{B}\right)-\int_{\Sigma} d^{n} x \dot{z}^{A} \frac{\delta G}{\delta z^{A}}\right. \\
& \left.-\partial_{t} G+\oint_{\partial \Sigma}\left(d^{n-1}\right)_{i}\left(V_{G}^{i}(z)-\Theta_{G}^{i}[\dot{z}]\right)\right\} \\
& =\int d t\left\{\frac{d}{d t}\left(\int_{\Sigma} d^{n} x \frac{1}{2} \sigma_{A B} z^{A} G^{B}-G\right)+\oint_{\partial \Sigma}\left(d^{n-1}\right)_{i} V_{G}^{i}(z)\right\}
\end{aligned}
$$

which means that $\delta_{G}$ is an external symmetry of the action. Between the second and the third line we used equation (3.25) and the fact that $\widehat{\delta} z^{A}=\delta_{H} z^{A}-\dot{z}^{A}$ preserves the internal boundary conditions. 
Theorem 3.5. The external symmetries of the action form an algebra. The associated generalized differentiable generators with the modified bracket form an extended representation of this algebra:

$$
\left\{G_{2}, G_{1}\right\}_{\zeta}=G_{[1,2]}+\oint_{\partial \Sigma}\left(d^{n-1} x\right)_{i} K_{1,2}^{i}(z)
$$

where the extra boundary term is invariant under all variations preserving the internal boundary conditions and satisfies

$$
\begin{gathered}
K_{1,2}^{i}=-K_{2,1}^{i} \\
\oint_{\partial \Sigma}\left(d^{n-1} x\right)_{i}\left(K_{[1,2], 3}^{i}+\delta_{3} K_{1,2}^{i}+\text { cyclic }\right)=0 .
\end{gathered}
$$

As before, this extension is in general non-central as it is based on the representation of the algebra of external symmetries on the boundary fields $\zeta$.

Proof. The modified poisson bracket of two generalized differentiable generators is a generalized differentiable generator. The only thing we need is to check the modified conservation law (3.25). Let's assume $F$ and $G$ generate external symmetries of the action, we get

$$
\begin{aligned}
\partial_{t}\{F, G\}_{\zeta}+\left\{\{F, G\}_{\zeta}, H\right\}_{\zeta} & =\left\{\partial_{t} F+\{F, H\}_{\zeta}, G\right\}_{\zeta}+\left\{F, \partial_{t} G+\{G, H\}_{\zeta}\right\}_{\zeta} \\
& =\left\{\oint_{\partial \Sigma}\left(d^{n-1} x\right)_{i} V_{F}^{i}, G\right\}_{\zeta}+\left\{F, \oint_{\partial \Sigma}\left(d^{n-1} x\right)_{i} V_{G}^{i}\right\}_{\zeta} \\
& =\oint_{\partial \Sigma}\left(d^{n-1}\right)_{i}\left(\delta_{G} V_{F}^{i}-\delta_{F} V_{G}^{i}\right) .
\end{aligned}
$$

The boundary term in the last line is zero under an arbitrary variation preserving the internal boundary conditions.

We proved that the external symmetries of the action form an algebra. Let's consider $G_{1}, G_{2}$ and $G_{[1,2]}$ respectively the generators of the external symmetries of the action $\delta_{1}, \delta_{2}$ and $\delta_{[1,2]}$. As we showed, $G_{[1,2]}$ and $\left\{G_{2}, G_{1}\right\}$ generate the same transformation. Their difference is a generalized differentiable generator producing a zero variation: it is a boundary term invariant under all variations preserving the internal boundary conditions. We have

$$
\begin{gathered}
\left\{G_{2}, G_{1}\right\}_{\zeta}=G_{[1,2]}+\oint_{\partial \Sigma}\left(d^{n-1} x\right)_{i} K_{1,2}^{i}(z), \\
\delta \oint_{\partial \Sigma}\left(d^{n-1} x\right)_{i} K_{1,2}^{i}(z)=0
\end{gathered}
$$

for all variations $\delta z^{A}$ preserving the internal boundary conditions. The antisymmetry of the poisson bracket means that

$$
K_{1,2}^{i}=-K_{2,1}^{i}
$$


If we have a third external symmetry $\delta_{3}$ then the Jacobi identity for the modified poisson bracket gives the cyclic identity:

$$
\oint_{\partial \Sigma}\left(d^{n-1}\right)_{i}\left[K_{1,[2,3]}^{i}+\delta_{3} K_{1,2}^{i}+\text { cyclic }\right]=0
$$

\subsection{Example: scalar field}

In this section, we will use the theory of a single scalar field as an example for the results we presented in the previous section. The hamiltonian action is given by

$$
S[\phi, \pi]=\int d t \int_{\Sigma} d^{3} x\left\{\pi \dot{\phi}-\frac{1}{2}\left(\frac{1}{\sqrt{g}} \pi^{2}+\sqrt{g} g^{i j} \partial_{i} \phi \partial_{j} \phi\right)\right\},
$$

where $g_{i j}$ is the metric on $\Sigma$. We will consider $\Sigma$ a ball in $\mathbb{R}^{n}$ with its boundary being a $n-1$ sphere: $\partial \Sigma=S_{n-1}$. We will take the metric to be flat and use spherical coordinates $x^{i}=r, x^{A}$ :

$$
g_{i j} d x^{i} d x^{j}=d r^{2}+r^{2} \gamma_{A B} d x^{A} d x^{B} .
$$

The covariant derivative associated to $g_{i j}$ will be denoted $D_{i}$, in particular, we will have $D_{i} \pi=\partial_{i} \pi-\Gamma_{i j}^{j} \pi$ as $\pi$ is a density. We will use Dirichlet boundary conditions:

$$
\chi_{I}(\phi, \pi)=\phi,\left.\quad \chi_{I}(\phi, \pi)\right|_{\partial \Sigma}=\bar{\phi}\left(t, x^{A}\right)
$$

To have smooth solutions, we also need to impose the external boundary conditions (3.5). When the boundary field $\bar{\phi}$ is fixed, they become:

$$
\left.\frac{1}{\sqrt{g}} \pi\right|_{\partial \Sigma}=\frac{d}{d t} \bar{\phi},\left.\quad\left(D^{i} D_{i}\right)^{k} \phi\right|_{\partial \Sigma}=\frac{d^{2 k}}{d t^{2 k}} \bar{\phi},\left.\quad\left(D^{i} D_{i}\right)^{k}\left(\frac{1}{\sqrt{g}} \pi\right)\right|_{\partial \Sigma}=\frac{d^{2 k+1}}{d t^{2 k+1}} \bar{\phi}
$$

for all integers $k>0$. The laplacian in $n$ dimensions $D^{i} D_{i}$ can be decomposed into its radial and angular part. Doing this, the external boundary conditions become boundary conditions on some combinations of radial derivatives of the dynamical fields.

A scalar field in $\mathbb{R}^{n+1}$ has the full Poincare symmetry. If we restrict the theory to $\Sigma$, this symmetry is broken to the subgroup preserving the boundary conditions $\bar{\phi}\left(t, x^{A}\right)$. For general values of $\bar{\phi}$, the resulting symmetry group is trivial.

Let's now consider the external symmetries we defined in the previous section and see what subalgebra of Poincaré is preserved. Poincaré transformations take the form

$$
\delta_{\xi} \phi=\frac{\xi^{\perp}}{\sqrt{g}} \pi+\xi^{i} \partial_{i} \phi, \quad \delta_{\xi} \pi=\sqrt{g} g^{i j} D_{i}\left(\xi^{\perp} \partial_{j} \phi\right)+\partial_{i}\left(\xi^{i} \pi\right)
$$


where $\xi^{i}-t D^{i} \xi^{\perp}$ is a time independent killing vector of $g_{i j}$ and $\xi^{\perp}$ satisfies $\partial_{t} \xi^{\perp}=0$ and $D_{i} D_{j} \xi^{\perp}=0$. One can easily check that those combine into $\xi^{\mu}=\left(\xi^{\perp}, \xi^{i}\right)$ to form a killing vector of Minkowski $d s^{2}=-d t^{2}+g_{i j} d x^{i} d x^{j}$. As $\delta_{\xi}$ is a symmetry of the action, we have $\left[d_{t}-\delta_{t}, \delta_{\xi}\right]=0$. This leads to:

$$
\begin{aligned}
\delta_{\xi} \chi_{I} & =\frac{\xi^{\perp}}{\sqrt{g}} d_{t} \bar{\phi}+\xi^{A} \partial_{A} \bar{\phi}+\xi^{r} \partial_{r} \phi+\xi^{A} \partial_{A}\left(\chi_{I}-\bar{\phi}\right)+\frac{\xi^{\perp}}{\sqrt{g}}\left(\delta_{t}-d_{t}\right) \chi_{I}, \\
\delta_{\xi} \chi_{E}^{k} & =\left(d_{t}-\delta_{t}\right)^{k} \delta_{\xi} \chi_{I}, \\
& =\xi^{r}\left(d_{t}-\delta_{t}\right)^{k} \partial_{r} \phi+\xi^{A} \partial_{A}\left[\left(d_{t}-\delta_{t}\right)^{k} \chi_{I}\right]-\frac{\xi^{\perp}}{\sqrt{g}}\left(d_{t}-\delta_{t}\right)^{k+1} \chi_{I} .
\end{aligned}
$$

The transformation $\delta_{\xi}$ satisfy the boundary conditions of an external symmetry (3.10) if and only if $\left.\xi^{r}\right|_{\partial \Sigma}=0$. From the Poincaré transformations, only linear combinations of time translation and rotations satisfy those two conditions. This seems natural as they are the only transformations preserving $\Sigma$. The associated generalized differential generator is

$$
\begin{gathered}
G\left[\xi^{\perp}, \xi^{i}\right]=\int_{\Sigma} d^{n} x\left(\xi^{\perp} \mathcal{H}_{\perp}+\xi^{A} \mathcal{H}_{A}\right) \\
\mathcal{H}_{\perp}=\frac{1}{2}\left(\frac{1}{\sqrt{g}} \pi^{2}+\sqrt{g} g^{i j} \partial_{i} \phi \partial_{j} \phi\right), \quad \mathcal{H}_{i}=\pi \partial_{i} \phi
\end{gathered}
$$

with $\xi^{\perp}$ a constant and $\xi^{A}$ a killing vector of the $n-1$ sphere. A variation of the fields $\delta$ preserving only the external boundary conditions $\left.\delta \chi_{E}\right|_{\partial \Sigma}=0$ leads to

$$
\begin{gathered}
\delta G\left[\xi^{\perp}, \xi^{i}\right]=\int_{\Sigma} d^{n} x\left(\frac{\delta G}{\delta \phi} \delta \phi+\frac{\delta G}{\delta \pi} \delta \pi\right)+\oint_{\partial \Sigma}\left(d^{n-1} x\right)_{r} \sqrt{g} \xi^{\perp} D^{r} \phi \delta \bar{\phi} \\
\Rightarrow \quad \Theta_{\xi}^{r}[\delta \bar{\phi}]=\sqrt{g} \xi^{\perp} D^{r} \phi \delta \bar{\phi} .
\end{gathered}
$$

The poisson bracket of two generators of the form (3.55) is given by

$$
\begin{aligned}
\left\{G\left[\xi^{\perp}, \xi^{i}\right], G\left[\eta^{\perp}, \eta^{i}\right]\right\}=\int_{\Sigma} d^{n} x\{ & \left.\left(\xi^{B} \partial_{B} \eta^{A}-\eta^{B} \partial_{B} \xi^{A}\right) \mathcal{H}_{A}\right\} \\
& +\oint_{\partial \Sigma}\left(d^{n-1}\right)_{r} \sqrt{g}\left(\eta^{\perp} \xi^{A}-\xi^{\perp} \eta^{A}\right) D^{r} \phi \partial_{A} \phi .
\end{aligned}
$$

We see that the poisson bracket produces an extra boundary term. However, as expected, this boundary term is killed if we use the modified bracket (3.17):

$$
\left\{G\left[\xi^{\perp}, \xi^{i}\right], G\left[\eta^{\perp}, \eta^{i}\right]\right\}_{\zeta}=\int_{\Sigma} d^{n} x\left\{\left(\xi^{B} \partial_{B} \eta^{A}-\eta^{B} \partial_{B} \xi^{A}\right) \mathcal{H}_{A}\right\} .
$$

The algebra closes without extension. The hamiltonian being given by $H=G[1,0]$ leads to

$$
\partial_{t} G\left[\xi^{\perp}, \xi^{i}\right]+\left\{G\left[\xi^{\perp}, \xi^{i}\right], H\right\}_{\zeta}=0
$$


which proves that these transformations are external symmetries of the theory.

In this section, we have restricted our analysis to the Poincaré transformations for clarity. On top of considering the boundary conditions as sources, we also could have treated the metric as a source. The external symmetries would then include all diffeomorphisms preserving the form of the boundary.

\section{Surface charges for gauge theories}

Before studying the external symmetries of gauge field theories, we will spend some time studying symmetries and conserved charges for gauge theories with a finite number of degrees of freedom.

\subsection{Symmetries of gauge theories}

The theories we will work with are of the form:

$$
S\left[z^{A}, \lambda^{a}\right]=\int d t\left(\frac{1}{2} z^{A} \sigma \dot{z}^{B}-h(z)-\lambda^{a} \phi_{a}\right),
$$

where $\phi_{a}$ are first-class constraints and $h$ is a first-class function. The symmetries and associated conserved quantities of this class of theories are studied in exercise 3.24 of [10]. We will now review some of the results obtained in this reference and introduce some new concepts that will be needed in section 4.2

As in [10], let's consider a transformation of the form

$$
\delta_{G} z^{A}=Z^{A}(t, z, \lambda, \dot{\lambda}, \ldots, \stackrel{(k)}{\lambda}), \quad \delta_{G} \lambda^{a}=\Lambda^{a}(t, z, \lambda, \dot{\lambda}, \ldots, \stackrel{(k)}{\lambda}) .
$$

It is a symmetry of the action if and only if there exists a generator $G(t, z, \lambda, \dot{\lambda}, \ldots, \stackrel{(k)}{\lambda})$ such that

$$
\begin{gathered}
Z^{A}=\sigma^{A B} \frac{\partial}{\partial z^{B}} G \\
\frac{D}{D t} G+\{G, H\}=\Lambda^{a} \phi_{a}
\end{gathered}
$$

where

$$
\frac{D}{D t}=\frac{\partial}{\partial t}+\sum_{l=0} \lambda^{a} \frac{\partial}{\partial \lambda^{a}} \text { and } H=h+\lambda^{a} \phi_{a} .
$$

From (4.4), one can show that the dependence in $\lambda$ of the generator $G$ is proportional to the constraints:

$$
G(t, z, \lambda, \dot{\lambda}, \ldots, \stackrel{(k)}{\lambda})=\bar{G}(t, z)+g^{a}(t, z, \lambda, \dot{\lambda}, \ldots, \stackrel{(k)}{\lambda}) \phi_{a} .
$$


The conservation of $\bar{G}$ then takes the usual form:

$$
\frac{\partial}{\partial t} \bar{G}+\{\bar{G}, H\} \approx 0
$$

where $\approx$ denotes the equality on the constraint surface. Because of this, when studying symmetries of gauge theories, we usually restrict ourselves to cases for which both $Z^{A}$ and $G$ are independent of $\lambda$ as the potential dependence in the lagrange multipliers can always be absorbed by a gauge transformation.

When studying gauge field theories in the next section, this restriction might lead to problems. In some cases, the boundary conditions can create a link between the two kind of fields: $z^{A}$ and $\lambda^{a}$. In order to deal with this it will be easier to allow an explicit dependence on $\lambda^{a}$ in the variation of the canonical variables $\delta_{G} z^{A}$. Because of this, we will spend the rest of this section studying the algebra of the generators associated to symmetries of the form (4.2).

The first observation is that these symmetries don't form a closed subalgebra. The problem is that the commutator of two symmetries given by

$$
\begin{aligned}
& {\left[\delta_{1}, \delta_{2}\right] z^{A} }=Z_{1} \frac{\partial}{\partial z^{B}} Z_{2}^{A}+\sum_{l=0} d_{t}^{l} \Lambda_{1}^{b} \frac{\partial}{(l)} Z_{2}^{A}-(1 \leftrightarrow 2) \\
& {\left[\delta_{1}, \delta_{2}\right] \lambda^{a} }=Z_{1} \frac{\partial}{\partial z^{B}} \Lambda_{2}^{a}+\sum_{l=0} d_{t}^{l} \Lambda_{1}^{b} \frac{\partial}{\partial \lambda^{b}} \Lambda_{2}^{a}-(1 \leftrightarrow 2) \\
& \partial \lambda^{b}
\end{aligned}
$$

contains explicit dependence in the time derivative of $z^{A}$ through the terms $d_{t}^{l} \Lambda^{a}$. The way out is that we are studying equivalence classes of symmetries where two symmetries are equivalent if their difference is a trivial symmetry:

$$
\delta_{1} \sim \delta_{2} \Leftrightarrow\left\{\begin{array}{c}
\delta_{1} z^{A}-\delta_{2} z^{A}=M^{A B}\left(\frac{\delta L}{\delta z^{B}}\right)-M^{\dagger b A}\left(\frac{\delta L}{\delta \lambda^{b}}\right), \\
\delta_{1} \lambda^{a}-\delta_{2} \lambda^{a}=M^{a B}\left(\frac{\delta L}{\delta z^{B}}\right)+M^{a b}\left(\frac{\delta L}{\delta \lambda^{b}}\right)
\end{array}\right.
$$

with

$$
\begin{aligned}
& M \cdot \cdot(F)=\sum_{l=0}^{k} M_{l} \cdot d_{t}^{l} F, \quad M^{\dagger \cdot \cdot}(F)=\sum_{l=0}^{k}\left(-d_{t}\right)^{l}\left(M_{l} \cdot F\right), \\
& M^{\dagger A B}=-M^{B A}, \quad M^{\dagger a b}=-M^{b a} .
\end{aligned}
$$

Two equivalent symmetries will lead to conserved quantities that are equal on the equa- 
tions of motion. One can show that: $\left[\delta_{1}, \delta_{2}\right] \sim \delta_{[1,2]}$ where

$$
\begin{aligned}
\delta_{[1,2]} Z^{A}= & Z_{1} \frac{\partial}{\partial z^{B}} Z_{2}^{A}+\sum_{l=0} \delta_{t}^{l} \Lambda_{1}^{b} \frac{\partial}{(l)} Z_{2}^{A} \\
& \quad+\sum_{l=1} \sum_{i=0}^{l-1} \sigma^{A B} \frac{\partial}{\partial z^{B}}\left(\delta_{t}^{i} \Lambda_{1}^{b}\right)\left(-\delta_{t}\right)^{l-i-1}\left(\begin{array}{c}
\phi_{a} \frac{\partial}{(l)} \Lambda_{2}^{a} \\
\partial \lambda^{b}
\end{array}\right)-(1 \leftrightarrow 2), \\
\delta_{[1,2]} \lambda^{a}= & Z_{1} \frac{\partial}{\partial z^{B}} \Lambda_{2}^{a}+\sum_{l=0} \delta_{t}^{l} \Lambda_{1}^{b} \frac{\partial}{(l)} \Lambda_{2}^{a}-(1 \leftrightarrow 2) \\
\delta_{t} F= & \frac{D}{D t} F+\{F, H\} .
\end{aligned}
$$

The transformation $\delta_{[1,2]}$ is a symmetry of the action of the form (4.2) with the following generator:

$$
G_{[1,2]}=\left\{G_{2}, G_{1}\right\}+\sum_{l=0}\left(\begin{array}{c}
\delta_{t}^{l} \Lambda_{1}^{a} \frac{\partial}{\partial \lambda^{a}} G_{2}-\delta_{t}^{l} \Lambda_{2}^{a} \frac{\partial}{\partial \lambda^{a}} G_{1}
\end{array}\right) .
$$

At $t$ fixed, one can treat $\stackrel{(i)}{\lambda^{a}}$ as independent variables. If we define:

$$
\widetilde{\delta}_{G} z^{A}=Z_{G}^{A}, \quad \widetilde{\delta}_{G} \stackrel{(i)}{\lambda}^{a}=\delta_{t}^{i} \Lambda_{G}^{a}, \quad \forall i
$$

the above results can be rewritten:

$$
\begin{aligned}
\delta_{[1,2]} \lambda^{a} & =\widetilde{\delta}_{1} \Lambda_{2}^{a}-\widetilde{\delta}_{2} \Lambda_{1}^{a}, \\
G_{[1,2]} & =\left\{G_{2}, G_{1}\right\}^{g},
\end{aligned}
$$

where

$$
\begin{aligned}
\left\{G_{1}, G_{2}\right\}^{g} & \equiv\left\{G_{1}, G_{2}\right\}+\widetilde{\delta}_{2}^{\lambda} G_{1}-\widetilde{\delta}_{1}^{\lambda} G_{2} \\
& =\widetilde{\delta}_{2} G_{1}-\widetilde{\delta}_{2} G_{1}-\left\{G_{1}, G_{2}\right\} .
\end{aligned}
$$

The notation $\widetilde{\delta}^{\lambda}$ denotes the part of the variation only hitting the dependence in $\lambda^{a}$ and its time derivatives. Defining $\delta_{H} \lambda^{a}=\dot{\lambda}^{a}$, the conservation condition (4.4) becomes

$$
\frac{\partial}{\partial t} G+\{G, H\}^{g}=0
$$

These results are very similar to what we obtained when studying external sources in section 2. However, there are a few differences. The first one is that the transformation of the lagrange multipliers in (4.2) can depend on the canonical variables. The second one is that the bracket induced on the couples $\left(G, \delta_{G}^{\lambda}\right)$ does not satisfy the Jacobi identity. If we define:

$$
\begin{gathered}
{\left[\left(G_{1}, \delta_{1}^{\lambda}\right),\left(G_{2}, \delta_{2}^{\lambda}\right)\right] \equiv\left(\left\{G_{2}, G_{1}\right\}^{g}, \delta_{[1,2]}^{\lambda}\right)} \\
\widetilde{\delta}_{[1,2]} \stackrel{(i)}{\lambda}^{a}=\delta_{t}^{i}\left(\widetilde{\delta}_{1} \Lambda_{2}^{a}-\widetilde{\delta}_{2} \Lambda_{2}^{a}\right)
\end{gathered}
$$


then

$$
\left[\left[\left(G_{1}, \delta_{1}^{\lambda}\right),\left(G_{2}, \delta_{2}^{\lambda}\right)\right],\left(G_{3}, \delta_{3}^{\lambda}\right)\right]+c y c l=\left(G_{J}, \delta_{J}^{\lambda}\right)
$$

where

$$
G_{J}=\sum_{l=0}\left(\left[\widetilde{\delta}_{2}, \delta_{t}^{i}\right] \Lambda_{1}^{a}-\left[\widetilde{\delta}_{1}, \delta_{t}^{i}\right] \Lambda_{2}^{a}\right) \frac{\partial}{\partial \lambda^{a}} G_{3}+c y c l .
$$

If $\left(G_{3}, \delta_{3}^{\lambda}\right)=\left(H, \delta_{H}^{\lambda}\right)$ then this expression simplifies to $G_{J}=0$. This is another way of saying that, if both $\left(G_{1}, \delta_{1}^{\lambda}\right)$ and $\left(G_{2}, \delta_{2}^{\lambda}\right)$ generate symmetries of the action, then $\left[\left(G_{1}, \delta_{1}^{\lambda}\right),\left(G_{2}, \delta_{2}^{\lambda}\right)\right]$ also generates a symmetry of the action. If all three couples $\left(G_{1}, \delta_{1}^{\lambda}\right)$, $\left(G_{2}, \delta_{2}^{\lambda}\right)$ and $\left(G_{3}, \delta_{3}^{\lambda}\right)$ generate symmetries of the action, we have $G_{J} \approx 0$ as expected. In the case where the generators $G_{1}$ and $G_{2}$ are independent of the lagrange multipliers, the associated transformations $\delta_{1}^{\lambda}$ and $\delta_{2}^{\lambda}$ don't matter and we have

$$
\left\{G_{1}, G_{2}\right\}^{g}=\left\{G_{1}, G_{2}\right\}
$$

\subsection{Gauge field theories}

This section is dedicated to the generalisation of the results of section 3 to gauge field theories. We will see how our previous analysis can solve some integrability problems in the definition of surface charges associated to gauge transformations. The main idea will be to combine the results obtained in the previous subsection with those obtained in section 3.1 .

We will consider gauge theories of the form:

$$
\begin{gathered}
S[z, \lambda]=\int d t\left\{\int_{\Sigma} d^{n} x \frac{1}{2} z^{A} \sigma_{A B} \dot{z}^{B}-H[z, \lambda]\right\}, \\
H[z, \lambda]=\int_{\Sigma} d^{n} x\left(h(z)+\lambda^{a} \phi_{a}\right)+\oint_{\partial \Sigma}\left(d^{n-1} x\right)_{i} b_{H}^{i}(z, \lambda),
\end{gathered}
$$

where $\phi_{a}$ are first-class constraints and $h$ is a first-class hamiltonian. In general, both the dynamical fields $z^{A}$ and the lagrange multipliers $\lambda^{a}$ will have non-trivial internal boundary conditions:

$$
\left.\chi_{I}^{\alpha}(z, \lambda)\right|_{\partial \Sigma}=\zeta^{\alpha} .
$$

On top of these, we will impose the equations of motion and all their derivatives on the boundary:

$$
\left.\partial_{(i)} \phi_{a}\right|_{\partial \Sigma}=0,\left.\quad \partial_{(i)}\left(\dot{z}^{A}-\sigma^{A B} \frac{\delta H}{\delta z^{B}}\right)\right|_{\partial \Sigma}=0 .
$$

This requirement is stronger than the one imposed in section 3.1 as the smoothness conditions are automatically satisfied when the EOM are imposed on the boundary. Imposing all equations of motion on the boundary may seem like a very strong requirement, however, as we will only be concerned by symmetries, it will not restrict our analysis. 
The conditions (4.31) will be referred as the external boundary conditions. We will also assume the same regularity requirement as in section 3.1 for the variations: the set of variations preserving the internal boundary conditions

$$
\left.\delta \chi_{E}\right|_{\partial \Sigma}=0,\left.\quad \delta \chi_{I}^{\alpha}\right|_{\partial \Sigma}=0 \quad \forall(z, \lambda) \text { s.t. }\left.\chi_{E}\right|_{\partial \Sigma}=0
$$

when evaluated on a specific value of $\zeta^{\alpha}$ is equal to the set

$$
\left.\delta \chi_{E}\right|_{\partial \Sigma}=0,\left.\quad \delta \chi_{I}^{\alpha}\right|_{\partial \Sigma}=0 \quad \forall(z, \lambda) \text { s.t. }\left.\chi_{E}\right|_{\partial \Sigma}=0,\left.\quad \chi_{I}^{\alpha}\right|_{\partial \Sigma}=\zeta^{\alpha}
$$

We will require the total hamiltonian $H$ to have a well defined variation: for all variations $\delta$ preserving the internal boundary conditions, we have

$$
\delta H=\int_{\Sigma} d^{n} x\left(\frac{\delta H}{\delta z^{A}} \delta z^{A}+\phi_{a} \delta \lambda^{a}\right) .
$$

Let's consider a couple $\left(G, \delta_{G}^{\lambda}\right)$ such that

$$
\begin{gathered}
G[t, z,, \lambda, \dot{\lambda}, \ldots, \stackrel{(k)}{\lambda}], \quad \delta_{G}^{\lambda} \lambda^{a}=\Lambda^{a}(t, z, \lambda, \dot{\lambda}, \ldots, \stackrel{(k)}{\lambda}), \\
{\left.\left[\delta_{G}, \delta\right] \chi_{I}^{\alpha}\right|_{\partial \Sigma}=0, \quad \delta G=\int_{\Sigma} d^{n} x\left(\frac{\delta G}{\delta z^{A}} \delta z^{A}+\sum_{l=0} \frac{\delta G}{\delta \lambda^{a}} \delta \stackrel{(l)}{\lambda}^{a}\right),}
\end{gathered}
$$

for all variation $\delta$ preserving the internal boundary conditions. Using arguments similar to those used in section 3.1, one can prove:

Theorem 4.1. A couple $\left(G, \delta_{G}^{\lambda}\right)$ of the form (4.35) satisfying (4.36) is an external symmetry of the action if and only if there exists a boundary term $\oint V_{G}$ such that:

$$
\begin{gathered}
\frac{D}{D t} G+\{G, H\}_{\zeta}-\delta_{G}^{\lambda} H=\oint_{\partial \Sigma}\left(d^{n-1} x\right)_{i} V_{G}^{i}(z, \lambda, \dot{\lambda}, \ldots) \\
Z^{A}=\sigma^{A B} \frac{\delta G}{\delta z^{B}}, \quad \frac{D}{D t}=\frac{\partial}{\partial t}+\sum_{l=0} \partial_{(i)}{\stackrel{(l+1)}{\lambda^{a}} \frac{\partial}{\partial \lambda^{a}{ }_{(i)}}}^{(l)}
\end{gathered}
$$

with

$$
\delta \oint_{\partial \Sigma}\left(d^{n-1} x\right)_{i} V_{G}^{i}(z, \lambda, \dot{\lambda}, \ldots)=0,
$$

for all variations $\delta$ preserving the internal boundary conditions.

Remark that, because the transformations we are studying are locally symmetries of the equations of motion, the external boundary conditions are always preserved. This theorem has an interesting corollary which is the field theory equivalent of equation (4.6): 
Corollary 4.2. If a couple $\left(G, \delta_{G}^{\lambda}\right)$ of the form (4.35) satisfying (4.36) is an external symmetry of the action then

$$
\frac{\delta G}{\delta \lambda^{a}} \approx 0, \quad \forall l
$$

Proof. Using

$$
\delta_{t}=\frac{D}{D t}+\partial_{(i)}\left(\sigma^{A B} \frac{\delta H}{\delta z^{B}}\right) \frac{\partial^{S}}{\partial z_{(i)}^{A}}=\frac{\partial}{\partial t}+\delta_{H}, \quad \delta_{H} \lambda^{a}=\dot{\lambda}^{a},
$$

the conservation equation (4.37) can be written

$$
\delta_{t} G-\delta_{G}^{\lambda} H=\oint_{\partial \Sigma} V_{G}
$$

For $l>0$, if $\delta^{l}$ is an arbitrary bulk variation of $\lambda^{a}$ only, we get

$$
\delta_{t} \delta^{l} G+\delta^{l}{\stackrel{(l)}{\lambda^{a}}}^{\frac{\delta G}{(l-1)}}-\delta^{l} \Lambda^{a} \phi_{a}=0,
$$

up to a boundary term. Because $\delta_{t} \phi_{a} \approx 0$, starting from $l=k+1$, we obtain all the identities (4.40) recursively.

The main difference compared to the previous section is that in this case, we cannot remove the dependence of $G$ in the lagrange multipliers due to the boundary conditions involving both $\lambda$ and $z$.

Let's now consider two couples $\left(G_{1}, \delta_{1}^{\lambda}\right)$ and $\left(G_{2}, \delta_{2}^{\lambda}\right)$ generating external symmetries of the action. The commutator of these two symmetries will contain dependences in the time derivatives of the dynamical variables $z^{A}$. As in section 4.1, we can remove them using trivial symmetry transformations to obtain $\delta_{[1,2]} \sim\left[\delta_{1}, \delta_{2}\right]$ with:

$$
\begin{aligned}
\delta_{[1,2]} \lambda^{a}= & \widetilde{\delta}_{1} \Lambda_{2}^{a}-\widetilde{\delta}_{2} \Lambda_{1}^{a}, \\
\delta_{[1,2]} z^{A}= & \widetilde{\delta}_{1} Z_{2}^{A} \\
& +\sum_{l=1} \sum_{m=0}^{l-1} \sigma^{A D} \frac{\delta}{\delta z_{(j)}^{D}}\left(\delta_{t}^{m} \Lambda_{1}^{b}\right)(-\partial)_{(j)}\left(-\delta_{t}\right)^{l-1-m}\left(\frac{\delta}{\delta \lambda^{b}}\left(\phi_{a} \Lambda_{2}^{a}\right)\right) \\
& -(1 \leftrightarrow 2),
\end{aligned}
$$

where

$$
\widetilde{\delta}_{G}=\partial_{(i)}\left(\sigma^{A B} \frac{\delta G}{\delta z^{B}}\right) \frac{\partial^{S}}{\partial z_{(i)}^{A}}+\sum_{l=0} \partial_{(i)}\left(\delta_{t}^{l} \Lambda_{G}^{a}\right) \frac{\partial^{S}}{\partial \lambda^{a}{ }_{(i)}},
$$

with $\delta_{t}$ defined in equation (4.41). Because $\delta_{t}-d_{t}$ is proportional to the EOM and we imposed them on the boundary, the transformations $\widetilde{\delta}_{1}, \widetilde{\delta}_{2}$ and $\delta_{[1,2]}$ all preserve the external 
boundary conditions. One can check that the dynamical part of the transformation $\delta_{[1,2]}$ is generated by

$$
G_{[1,2]}=\left\{G_{2}, G_{1}\right\}_{\zeta}+\widetilde{\delta}_{1}^{\lambda} G_{2}-\widetilde{\delta}_{2}^{\lambda} G_{1}=\widetilde{\delta}_{1} G_{2}-\widetilde{\delta}_{2} G_{1}-\left\{G_{2}, G_{1}\right\},
$$

where $\{,\}_{\zeta}$ is the bracket involving only $z^{A}$ defined in section 3.1

Theorem 4.3. If $\left(G_{1}, \delta_{1}^{\lambda}\right)$ and $\left(G_{2}, \delta_{2}^{\lambda}\right)$ generate external symmetries of the action, then their bracket $\left[\left(G_{1}, \delta_{1}^{\lambda}\right),\left(G_{2}, \delta_{2}^{\lambda}\right)\right]$ defined by

$$
\begin{gathered}
{\left[\left(G_{1}, \delta_{1}^{\lambda}\right),\left(G_{2}, \delta_{2}^{\lambda}\right)\right]=\left(\left\{G_{2}, G_{1}\right\}_{g}, \delta_{[1,2]}^{\lambda}\right),} \\
\left\{G_{2}, G_{1}\right\}_{g}=\widetilde{\delta}_{1} G_{2}-\widetilde{\delta}_{2} G_{1}-\left\{G_{2}, G_{1}\right\}, \\
\delta_{[1,2]}^{\lambda} \lambda^{a}=\widetilde{\delta}_{1} \Lambda_{2}^{a}-\widetilde{\delta}_{2} \Lambda_{1}^{a},
\end{gathered}
$$

satisfies (4.36) for all variations preserving the internal boundary conditions and generates an external symmetry of the action.

Using this bracket, the conservation condition (4.37) can be rewritten

$$
\frac{\partial}{\partial t}+\{G, H\}_{g}=\oint_{\partial \Sigma}\left(d^{n-1}\right)_{i} V_{G}^{i}
$$

Proof. The first condition of (4.36) is direct using the Jacobi identity for the variations. In appendix $\mathrm{B}$, we prove that for all variations $\delta$ preserving the internal boundary conditions, we have:

$$
\begin{aligned}
& \delta\left\{G_{2}, G_{1}\right\}_{g}=\delta z^{A} \frac{\delta}{\delta z^{A}}\left\{G_{2}, G_{1}\right\}_{g}
\end{aligned}
$$

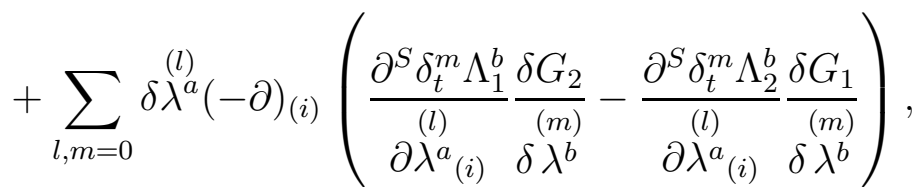

which is exactly equation 4.36).

In appendix $\mathrm{D}$, we prove that

$$
\left\{\left\{G_{2}, G_{1}\right\}_{g}, H\right\}_{g}=-\left\{\left\{G_{2}, H\right\}_{g}, G_{1}\right\}_{g}+\left\{\left\{G_{1}, H\right\}_{g}, G_{2}\right\}_{g} .
$$

Because both $\left(G_{1}, \delta_{1}^{\lambda}\right)$ and $\left(G_{2}, \delta_{2}^{\lambda}\right)$ generate symmetries of the action, they satisfy the conservation condition (4.51). Combining these identities with $\delta_{[1, H]}^{\lambda}=0$, we get

$$
\frac{\partial}{\partial t}\left\{G_{2}, G_{1}\right\}_{g}+\left\{\left\{G_{2}, G_{1}\right\}_{g}, H\right\}_{g}=\oint_{\partial \Sigma}\left(\widetilde{\delta}_{2} V_{1}-\widetilde{\delta}_{1} V_{2}\right)
$$

with

$$
\delta \oint_{\partial \Sigma}\left(\widetilde{\delta}_{2} V_{1}-\widetilde{\delta}_{1} V_{2}\right)=0
$$

for all $\delta$ preserving the internal boundary conditions. This proves that the bracket of two external symmetries is an external symmetry. 
A subset of the symmetries of the action are proper gauge transformations. These transformations generate the redundancy in the description of the theory. We will define them as:

Definition 4.4. The transformation $\delta_{\Gamma}$ generated by a couple $\left(\Gamma, \delta_{\Gamma}^{\lambda}\right)$ is a proper gauge transformation if it is an external symmetry preserving the internal boundary conditions $\left.\delta_{\Gamma} \chi_{I}^{\alpha}\right|_{\partial \Sigma}=0$ and if its generator satisfies:

$$
\Gamma \approx 0
$$

The requirement here is stronger than usual. The main difference is that we are treating all values of the boundary field $\zeta^{\alpha}$ at the same time. It is possible that the set of proper gauge transformation defined above, when evaluated for a specific value of $\zeta^{\alpha}$, is smaller than the set computed at fixed $\zeta^{\alpha}[11]$. In the following, we will assume that the set of proper gauge transformations defined here generates all proper gauge transformation for each value of the boundary field $\zeta^{\alpha}$. In other word, fixing these proper gauge transformations completely fixes the gauge freedom of each independent theory associated with the different values of the boundary conditions.

With the above definition, we obtain the expected result:

Theorem 4.5. If the transformation generated by a couple $\left(G, \delta_{G}^{\lambda}\right)$ is an external symmetry then $G$ is a first-class functional: for all $\left(\Gamma, \delta_{\Gamma}^{\lambda}\right)$ generating proper gauge transformations, the external symmetry generated by $\left[\left(G, \delta_{G}^{\lambda}\right),\left(\Gamma, \delta_{\Gamma}^{\lambda}\right)\right]$ is a proper gauge transformation.

Proof. Let's consider two couples $\left(G, \delta_{G}^{\lambda}\right)$ and $\left(\Gamma, \delta_{\Gamma}^{\lambda}\right)$ respectively generating an external symmetry and a proper gauge symmetry. By definition, as $\delta_{\Gamma}$ preserves the internal boundary conditions, the bracket $\left[\delta_{G}, \delta_{\Gamma}\right]$ will also preserve them. We have $\Gamma \approx 0$ for all values of $\zeta^{\alpha}$ : this implies $\delta_{G} \Gamma \approx 0$. As $\delta_{\Gamma}$ preserves the internal boundary conditions, we also have:

$$
\delta_{\Gamma} G-\{G, \Gamma\}=\int_{\Sigma} d^{n} x \sum_{l=0} \frac{\delta G}{\delta \lambda^{a}}{ }^{(l)} \delta \lambda^{a} \approx 0,
$$

using corollary 4.2. These two results combine to

$$
\{G, \Gamma\}_{g} \approx 0
$$

Another way of expressing this result is that the sub-algebra of proper gauge transformations forms an ideal. The Jacobi identity for the modified bracket defined in (4.48) is not valid (see appendix (D). However, we still have 
Theorem 4.6. The bracket induced on the quotient of the couples $\left(G, \delta_{G}^{\lambda}\right)$ generating external symmetries by the couples generating proper gauge transformations forms a representation of the algebra obtained form the quotient of external symmetries by proper gauge transformations.

Proof. Let's consider three couples $\left(G_{1}, \delta_{1}^{\lambda}\right),\left(G_{2}, \delta_{2}^{\lambda}\right)$ and $\left(G_{3}, \delta_{3}^{\lambda}\right)$ generating external symmetries. We proved in appendix $\mathrm{D}$ that the cyclic combination

$$
\left[\left[\left(G_{1}, \delta_{1}^{\lambda}\right),\left(G_{2}, \delta_{2}^{\lambda}\right)\right],\left(G_{3}, \delta_{3}^{\lambda}\right)\right]+\operatorname{cyclic}=\left(G_{J}, \delta_{J}^{\lambda}\right)
$$

satisfies $G_{J} \approx 0$. Due to the Jacobi identity of transformations, we know that $\delta_{J}$ differs form zero by a trivial symmetry. Because we imposed all equations of motion as boundary conditions, the transformation $\delta_{J}$ preserves the internal boundary conditions. This proves that $\left(G_{J}, \delta_{J}^{\lambda}\right)$ generates a proper gauge transformation. From this, the theorem follows easily.

Let's consider two couples $\left(G_{1}, \delta_{1}^{\lambda}\right)$ and $\left(G_{2}, \delta_{2}^{\lambda}\right)$ generating external symmetries as well as $\left(G_{[1,2]}, \delta_{[1,2]}^{\lambda}\right)$ a generator of the external symmetry $\delta_{[1,2]} \sim\left[\delta_{1}, \delta_{2}\right]$. We showed that $\left(\left\{G_{2}, G_{1}\right\}_{g}, \delta_{[1,2]}^{\lambda}\right)$ also generates $\delta_{[1,2]}$. This means that both functional differ by a functional that is in the kernel of $\frac{\delta}{\delta z^{A}}$ :

$$
\begin{gathered}
\left\{G_{2}, G_{1}\right\}=G_{[1,2]}+K_{1,2}, \\
K_{1,2}=\int_{\Sigma} d^{n} x K_{1,2}^{b u l k}(t, \lambda, \ldots)+\oint_{\Sigma}\left(d^{n-1} k\right)_{i} K_{1,2}^{i}(t, z, \lambda, \ldots) .
\end{gathered}
$$

Because of equation (4.40), the bulk part of the functional must be independent of the lagrange multipliers. What is left can be absorbed into the boundary term. Equation (4.36) then implies that $K_{1,2}$ can only depend on the boundary fields $\zeta$ :

$$
\delta K_{1,2}=\delta \oint_{\partial \Sigma}\left(d^{n-1} x\right)_{i} K_{1,2}^{i}=0
$$

for all variations $\delta$ preserving the internal boundary conditions. Using the previous results, we also get

Theorem 4.7. If $G_{i}$ forms a generating set of the algebra $\mathcal{G}$, we have in general

$$
\left[\left(G_{1}, \delta_{1}^{\lambda}\right),\left(G_{2}, \delta_{2}^{\lambda}\right)\right]=\left(G_{[1,2]}+K_{1,2}, \delta_{[1,2]}^{\lambda}\right)
$$

with $K_{1,2}$ antisymmetric and

$$
K_{[1,2], 3}+\delta_{3}^{j} K_{1,2}+\text { cyclic }=0 .
$$

As in the previous cases, the representation of the algebra of external symmetries has room for abelian extensions. 


\subsection{Link with the non-integrability of surface charge}

The method usually used to define charges for improper gauge transformations is the following (see [11] and references therein): after having chosen a set of boundary conditions for which the action is well defined, one computes the set of gauge transformations preserving the boundary conditions and then select the subset for which it is possible to define differentiable generators. In order to make the link with the existing literature easier, we will only consider transformations of the canonical variables that are independent of the lagrange multipliers.

The integrability problem appears in the last step. One has to solve an integrability condition to find the correct boundary term $k_{\epsilon}$ :

$$
\begin{gathered}
\delta \int_{\Sigma} d^{n} x \epsilon^{a} \phi_{a}=\int_{\Sigma} d^{n} x \frac{\delta \epsilon^{a} \phi_{a}}{\delta z^{A}} \delta z^{A}+\oint_{\partial \Sigma}\left(d^{n-1} x\right)_{i} \Theta_{\epsilon}^{i}[\delta z], \\
\delta \oint_{\partial \Sigma}\left(d^{n-1} x\right)_{i} k_{\epsilon}^{i}=-\oint_{\partial \Sigma}\left(d^{n-1} x\right)_{i} \Theta_{\epsilon}^{i}[\delta z]
\end{gathered}
$$

where the equalities are valid for all variations $\delta$ preserving the boundary conditions. If such a boundary term exists, the differentiable generator of the transformation $\delta_{\epsilon}$ associated to the gauge parameter $\epsilon$ is then

$$
G_{\epsilon}=\int_{\Sigma} d^{n} x \epsilon^{a} \phi_{a}+\oint_{\partial \Sigma}\left(d^{n-1} x\right)_{i} k_{\epsilon}^{i} .
$$

If this boundary term does not exists, the associated transformation is not canonical and it cannot be regarded as a symmetry of the action.

The usual way to solve this problem is to tighten the restrictions on the boundary. This reduces the set of variations in (4.66) and may help to define a suitable boundary term. However, doing this also reduces the set of gauge transformations preserving the boundary conditions and perhaps remove the transformations of interest.

The notion of external symmetry we introduced in this work brings another solution to this problem. The main idea is that the boundary conditions on the symmetries and the boundary conditions on the transformations used in the integrability conditions are different. Symmetries must only preserve the external boundary conditions and transform the boundary fields of the internal boundary conditions in an appropriate way whereas the transformations used in the variation condition (4.36) must preserve the stronger internal boundary conditions.

\section{Acknowledgements}

I would like to thank G. Barnich and P. Ritter for useful discussions. This work is founded by the fundecyt postdoctoral grant 3140125. The Centro de Estudios Científicos (CECs) 
is funded by the Chilean Government through the Centers of Excellence Base Financing Program of Conicyt.

\section{A Algebra for the poisson bracket with sources}

In this appendix, we will show that $\left(\{G, F\}_{j},\left[\delta_{F}^{j}, \delta_{G}^{j}\right]\right)$ satisfies

$$
\partial_{t}\{G, F\}_{j}+\left\{\{G, F\}_{j}, H\right\}_{j}=V_{\{G, F\}}
$$

We start by using the Jacobi identity on the second term:

$$
\begin{aligned}
\left\{\{G, F\}_{j}, H\right\}_{j} & =\left\{\{H, F\}_{j}, G\right\}_{j}+\left\{F,\{H, G\}_{j}\right\}_{j} \\
& =\left\{\partial_{t} F-V_{F}, G\right\}_{j}+\left\{F, \partial_{t} G-V_{G}\right\}_{j}
\end{aligned}
$$

Using the fact that both $\left(F, \delta_{F}^{j}\right)$ and $\left(G, \delta_{G}^{j}\right)$ are generators of symmetry. Using equation (2.29), we see that $\partial_{t} F-V_{F}$ is associated to the following operator acting on the sources:

$$
\begin{aligned}
\delta_{\partial_{t} F}^{j} j^{\alpha} & =\left[\delta_{F}^{j}, \delta_{H}^{j}\right] j^{\alpha} \\
& =\delta_{F}^{j} d_{t} j^{\alpha}-\delta_{t}^{j} J_{F}^{\alpha} \\
& =\partial_{t} J_{F}^{\alpha} .
\end{aligned}
$$

Now, we can expend equation (A.3) to

$$
\begin{aligned}
\left\{\{G, F\}_{j}, H\right\}_{j}= & \left\{\partial_{t} F-V_{F}, G\right\}+\delta_{G}^{j}\left(\partial_{t} F-V_{F}\right)-\delta_{\partial_{t} F}^{j} G \\
& \quad+\left\{F, \partial_{t} G-V_{G}\right\}+\delta_{\partial_{t} G}^{j} F-\delta_{F}^{j}\left(\partial_{t} G-V_{G}\right) \\
= & \partial_{t}\{F, G\}+\delta_{G}^{j}\left(\partial_{t} F-V_{F}\right)+\delta_{\partial_{t} G}^{j} F-\delta_{F}^{j}\left(\partial_{t} G-V_{G}\right)-\delta_{\partial_{t} F}^{j} G \\
= & \partial_{t}\{F, G\}+\partial_{t} \delta_{G}^{j} F-\partial_{t} \delta_{F}^{j} G-\delta_{G}^{j} V_{F}+\delta_{F}^{j} V_{G} \\
= & \partial_{t}\{F, G\}_{j}+\left(\delta_{F}^{j} V_{G}-\delta_{G}^{j} V_{F}\right),
\end{aligned}
$$

which is what we wanted with $V_{\{G, F\}}=\delta_{F}^{j} V_{G}-\delta_{G}^{j} V_{F}$. Between the second and the third line, we used the following identity

$$
\left[\partial_{t}, \delta_{G}^{j}\right]=\left[d_{t}-\delta_{t}^{j}-\dot{z}^{A} \frac{\partial}{\partial z^{A}}, \delta_{G}^{j}\right]=\left[-\delta_{t}^{j}, \delta_{G}^{j}\right]=\delta_{\partial_{t} G}^{j}
$$

\section{B Variation of the modified brackets}

In this appendix, we will study the behavior of our new brackets for field theories. This analysis applies to both the poisson bracket taking into account the boundary conditions defined in section 3 and the bracket of conserved quantities for gauge field theories defined in section 4.2 
Both bracket can be written as

$$
\{F, G\}^{\text {mod }}=\widetilde{\delta}_{G} F-\widetilde{\delta}_{F} G-\{F, G\}
$$

where the variations act on all the fields. The definition of $\widetilde{\delta}_{F, G}$ is given in section 4.2 In the non-gauge theory case, will assume that $F$ and $G$ are generalized differentiable functionals. In the gauge theory case, we will consider two couples $\left(F, \delta_{F}^{\lambda}\right)$ and $\left(G, \delta_{G}^{\lambda}\right)$ satisfying (4.36) and generating external symmetries of the action. If $\delta$ is a variation preserving the internal boundary conditions then $\left[\delta, \widetilde{\delta}_{G}\right]$ also preserves them. We have

$$
\begin{aligned}
\delta \widetilde{\delta}_{G} F & =\left[\delta, \widetilde{\delta}_{G}\right] F+\widetilde{\delta}_{G} \delta F \\
& =\delta Z_{G}^{A} \frac{\delta F}{\delta z^{A}}+\sum_{l=0} \delta \delta_{t}^{l} \Lambda_{G}^{a} \frac{\delta F}{\delta \lambda^{a}}+\delta z^{A} \widetilde{\delta}_{G} \frac{\delta F}{\delta z^{A}}+\sum_{l=0} \delta \lambda^{a} \widetilde{\delta}_{G} \frac{\delta F}{\delta \tilde{\lambda}^{a}}
\end{aligned}
$$

In the non-gauge theory case, the terms containing $\lambda$ are absent. Commuting $\widetilde{\delta}_{G}$ and $\frac{\delta}{\delta z^{A}}$, we get

$$
\widetilde{\delta}_{G} \frac{\delta F}{\delta z^{A}}=\frac{\delta}{\delta z^{A}} \widetilde{\delta}_{G} F-\delta z^{A}\left(-\partial_{i}\right)\left(\frac{\partial Z_{G}^{B}}{\partial z_{(i)}^{A}} \frac{\delta F}{\delta z^{B}}+\sum_{l=0} \frac{\partial \delta_{t}^{i} \Lambda_{G}^{b}}{\partial z_{(i)}^{A} \frac{\delta F}{\delta \lambda^{b}}}\right) .
$$

Using the fact that we imposed the constraints and all their derivatives to be zero on the boundary and corollary 4.2 , we also have

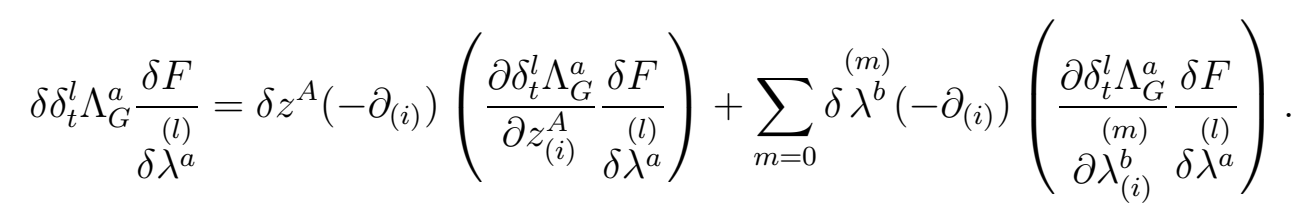

Lastly, the variation of the non-modified poisson bracket can be written as:

$$
\delta\{F, G\}=\delta Z_{G}^{A} \frac{\delta F}{\delta z^{A}}-\delta Z_{F}^{A} \frac{\delta G}{\delta z^{A}} .
$$

Combining everything, we get

$$
\begin{aligned}
\delta\{F, G\}^{m o d}=\delta z^{A} \frac{\delta}{\delta z^{A}}\left(\widetilde{\delta}_{G} F\right. & \left.-\widetilde{\delta}_{F} G-\{F, G\}\right) \\
& +\sum_{l, m=0} \delta \lambda^{b}(-\partial)_{(i)}\left(\begin{array}{c}
\frac{\partial \delta_{t}^{l} \Lambda_{G}^{a}}{(m)} \frac{\delta F}{(l)}-\frac{\partial \delta_{t}^{l} \Lambda_{F}^{a}}{\partial \lambda_{(i)}^{b}} \frac{\delta G}{\delta \lambda^{a}} \frac{(m)}{\partial \lambda_{(i)}^{b}} \delta \lambda^{a}
\end{array}\right)
\end{aligned}
$$

\section{Jacobi identity of the modified bracket}

In this appendix, we will use objects of the bi-variational formalism. We will follow the definitions, notations and properties introduced in appendix A of [8]. 
To prove the Jacobi identity, we will use the following expression for the modified poisson bracket of the n-forms $\widehat{F}$ and $\widehat{G}$ :

$$
\{\widehat{F}, \widehat{G}\}_{\zeta}=\delta_{G} \widehat{F}-d_{H} I_{F}^{n} \widehat{G}
$$

The corresponding bracket of the associated functionals $F=\int \widehat{F}$ and $G=\int \widehat{G}$ is

$$
\{F, G\}_{\zeta}=\int_{\Sigma}\{\widehat{F}, \widehat{G}\}_{\zeta}
$$

We can rewrite the Jacobi identity as

$$
\begin{aligned}
\left\{\widehat{F},\{\widehat{G}, \widehat{J}\}_{\zeta}\right\}_{\zeta}+\text { cycl }= & \left.\{\widehat{J}, \widehat{G}\}_{\zeta}, \widehat{F}\right\}_{\zeta}-\left\{\{\widehat{J}, \widehat{F}\}_{\zeta}, \widehat{G}\right\}_{\zeta} \\
& -\left\{\widehat{J},\{\widehat{G}, \widehat{F}\}_{\zeta}\right\}_{\zeta} \\
= & \delta_{F}\{\widehat{J}, \widehat{G}\}_{\zeta}-d_{H} I_{\{J, G\}}^{n} \widehat{F} \\
& -\delta_{G}\{\widehat{J}, \widehat{F}\}_{\zeta}+d_{H} I_{\{J, F\}}^{n} \widehat{G} \\
& -\delta_{\{G, F\}} \widehat{J}+d_{H} I_{J}^{n}\{\widehat{G}, \widehat{F}\}_{\zeta} \\
= & \delta_{F}\left(\delta_{G} \widehat{J}-d_{H} I_{J}^{n} \widehat{G}\right)-d_{H} I_{\{J, G\}}^{n} \widehat{F} \\
& -\delta_{G}\left(\delta_{F} \widehat{J}-d_{H} I_{J}^{n} \widehat{F}\right)+d_{H} I_{\{J, F\}}^{n} \widehat{G} \\
& -\delta_{\{G, F\}} \widehat{J}+d_{H} I_{J}^{n}\left(\delta_{F} \widehat{G}-d_{H} I_{G}^{n} \widehat{F}\right) \\
= & \left(\delta_{F} \delta_{G}-\delta_{G} \delta_{F}-\delta_{\{G, F\}}\right) \widehat{J} \\
& +d_{H}\left\{-\delta_{F} I_{J}^{n} \widehat{G}-I_{\{J, G\}}^{n} \widehat{F}+\delta_{G} I_{J}^{n} \widehat{F}\right. \\
& \left.+I_{\{J, F\}}^{n} \widehat{G}+I_{J}^{n}\left(\delta_{F} \widehat{G}-d_{H} I_{G}^{n} \widehat{F}\right)\right\} .
\end{aligned}
$$

From (3.18), we see that the first line gives zero, we are left with the boundary term. It can be simplified to

$$
\begin{aligned}
\text { Jacobi }=d_{H}\{ & -\delta_{F} I_{J}^{n} \widehat{G}-I_{[G, J]}^{n} \widehat{F}+\delta_{G} I_{J}^{n} \widehat{F} \\
& \left.+I_{[F, J]}^{n} \widehat{G}+I_{J}^{n} \delta_{F} \widehat{G}-\delta_{J} I_{G}^{n} \widehat{F}\right\} \\
=d_{H}\{ & -\left[\delta_{F}, I_{J}^{n}\right] \widehat{G}+I_{[F, J]}^{n} \widehat{G} \\
& +\left[\delta_{G}, I_{J}^{n}\right] \widehat{F}-I_{[G, J]}^{n} \widehat{F} \\
& \left.+I_{J}^{n} \delta_{G} \widehat{F}-\delta_{J} I_{G}^{n} \widehat{F}\right\} .
\end{aligned}
$$

Applying twice equations (A.40) of [8], we obtain

$$
J a c o b i=d_{H}\left\{T_{F}\left[J, \frac{\delta \widehat{G}}{\delta z}\right]-T_{G}\left[J, \frac{\delta \widehat{F}}{\delta z}\right]+I_{J}^{n} \delta_{G} \widehat{F}-\delta_{J} I_{G}^{n} \widehat{F}\right\} .
$$


We have two useful properties of these $T$ :

$$
\begin{aligned}
T_{Q_{1}}\left[Q_{2}, \frac{\delta \omega^{n}}{\delta z}\right] & =-W_{\frac{\delta \omega^{n}}{\delta z}}\left[Q_{2}, Q_{1}\right]+I_{Q_{2}}^{n}\left(Q_{1}^{B} \frac{\delta \omega^{n}}{\delta z^{B}}\right), \\
T_{F}\left[G, \frac{\delta \widehat{J}}{\delta z}\right] & =-W_{\frac{\delta \widehat{F}}{\delta z}}[G, J] .
\end{aligned}
$$

The first one is the equation (A.52) of [8]. The second one is just coming from the definition of $T$ and the properties of hamiltonian generators:

$$
\begin{aligned}
T_{F}\left[G, \frac{\delta \widehat{J}}{\delta z}\right] & =\left(\begin{array}{c}
|\mu|+|\rho|+1 \\
|\mu|+1
\end{array}\right) \partial_{(\mu)}\left(G^{A}(-\partial)_{(\rho)}\left(\frac{\partial^{S} F^{B}}{\partial z_{(\mu)(\rho) \nu}^{A}} \frac{\partial}{\partial d x^{\nu}} \frac{\delta \widehat{J}}{\delta z^{B}}\right)\right) \\
& =\left(\begin{array}{c}
|\mu|+|\rho|+1 \\
|\mu|+1
\end{array}\right) \partial_{(\mu)}\left(G^{A}(-\partial)_{(\rho)}\left(\sigma^{B C} \frac{\partial^{S}}{\partial z_{(\mu)(\rho) \nu}^{A}} \frac{\delta F}{\delta z^{C}} \frac{\delta J}{\delta z^{B}} d_{\nu}^{n-1} x\right)\right) \\
& =-\left(\begin{array}{c}
|\mu|+|\rho|+1 \\
|\mu|+1
\end{array}\right) \partial_{(\mu)}\left(G^{A}(-\partial)_{(\rho)}\left(\sigma^{C B} \frac{\delta J}{\delta z^{B}} \frac{\partial^{S}}{\partial z_{(\mu)(\rho) \nu}^{A}} \frac{\partial}{\partial d x^{\nu}} \frac{\delta\left(F d^{n} x\right)}{\delta z^{C}}\right)\right) \\
& =-\left(\begin{array}{c}
|\mu|+|\rho|+1 \\
|\mu|+1
\end{array}\right) \partial_{(\mu)}\left(G^{A}(-\partial)_{(\rho)}\left(J^{C} \frac{\partial^{S}}{\partial z_{(\mu)(\rho) \nu}^{A}} \frac{\partial}{\partial d x^{\nu}} \frac{\delta \vec{F}}{\delta z^{C}}\right)\right) \\
& =-W_{\frac{\delta \widehat{F}}{\delta z}}[G, J],
\end{aligned}
$$

using equation (A.51) of [8]. The next step is to apply (C.9) to the second term of (C.8) and (C.10) to the first term :

$$
\begin{aligned}
\text { Jacobi } & =d_{H}\left\{-W_{\frac{\delta \widehat{F}}{\delta z}}[J, G]+W_{\frac{\delta \widehat{F}}{\delta z}}[J, G]-I_{J}^{n}\left(G^{A} \frac{\delta \widehat{F}}{\delta z^{A}}\right)+I_{J}^{n} \delta_{G} \widehat{F}-\delta_{J} I_{G}^{n} \widehat{F}\right\} \\
& =d_{H}\left\{-I_{J}^{n}\left(\delta_{G} \widehat{F}-d_{H} I_{G} \widehat{F}\right)+I_{J}^{n} \delta_{G} \widehat{F}-\delta_{J} I_{G}^{n} \widehat{F}\right\} \\
& =0 .
\end{aligned}
$$

\section{Jacobi identity for gauge field theories}

This appendix is devoted to the computation of the cyclic identity of the modified bracket of conserved quantities in section 4.2. The form we will use is

$$
\{F, G\}_{g}=\{F, G\}_{\zeta}+\widetilde{\delta}_{G}^{\lambda} F-\widetilde{\delta}_{F}^{\lambda} G .
$$

Let's first prove a useful identity:

$$
\widetilde{\delta}^{\lambda}\{F, G\}_{\zeta}=\left\{\widetilde{\delta}^{\lambda} F, G\right\}_{\zeta}+\left\{F, \widetilde{\delta}^{\lambda} G\right\}_{\zeta}+\sum_{l=0} \partial_{(i)}\left[\delta_{F}^{z}\left(\delta_{t}^{l} \Lambda^{a}\right) \frac{\delta G}{\delta \lambda^{a}{ }_{(i)}}-\delta_{G}^{z}\left(\delta_{t}^{l} \Lambda^{a}\right) \frac{\delta F}{\delta \lambda^{a}{ }_{(i)}}\right]
$$

where $\delta^{\lambda} \lambda^{a}=\Lambda^{a}$. 
HAMILTONIAN SURFACE CHARGES USING EXTERNAL SOURCES.

Proof. We can write

$$
\{F, G\}_{\zeta}=\delta_{G}^{z} F-\delta_{F}^{z} G-\{F, G\}
$$

Using

$$
\begin{aligned}
\widetilde{\delta}^{\lambda} \delta_{G}^{z} F=\delta_{G}^{z} \widetilde{\delta}^{\lambda} F+\partial_{(i)}\left[\widetilde{\delta}^{\lambda}\left(\sigma^{A B} \frac{\delta G}{\delta z^{B}}\right) \frac{\delta F}{\delta z_{(i)}^{A}}+\sum_{l=0}\left(-\delta_{G}^{z} \delta_{t}^{l} \Lambda^{a}\right) \frac{\delta F}{\delta \lambda^{a}{ }_{(i)}}\right] \\
=\delta_{G}^{z} \widetilde{\delta}^{\lambda} F+\partial_{(i)}\left[\sigma^{A B} \frac{\delta}{\delta z^{B}}\left(\widetilde{\delta}^{\lambda} G\right) \frac{\delta F}{\delta z_{(i)}^{A}}-\sum_{l=0} \delta_{G}^{z}\left(\delta_{t}^{l} \Lambda^{a}\right) \frac{\delta F}{\delta \lambda^{(l)}{ }_{(i)}}\right] \\
+\partial_{(i)}\left[-\sum_{l=0} \sigma^{A B}(-\partial)_{(j)}\left(\frac{\partial \delta_{t}^{l} \Lambda^{a}}{\partial z_{(j)}^{B}} \frac{\delta G}{\delta \lambda^{(l)}}\right) \frac{\delta F}{\delta z_{(i)}^{A}}\right]
\end{aligned}
$$

with

$$
\widetilde{\delta}^{\lambda} \frac{\delta F}{\delta z^{A}} \sigma^{A B} \frac{\delta G}{\delta z^{B}}=\frac{\delta}{\delta z^{A}}\left(\widetilde{\delta}^{\lambda} F\right) \sigma^{A B} \frac{\delta G}{\delta z^{B}}-\sum_{l=0}(-\partial)_{(i)}\left(\frac{\partial \delta_{t}^{l} \Lambda^{a}}{\partial z_{(i)}^{A}} \frac{\delta F}{\delta \lambda^{a}}\right) \sigma^{A B} \frac{\delta G}{\delta z^{B}},
$$

we get

$$
\begin{aligned}
\widetilde{\delta}^{\lambda}\{F, G\}_{\zeta}= & \delta_{G}^{z} \widetilde{\delta}^{\lambda} F+\delta_{\tilde{\delta}^{\lambda} G}^{z} F-\left\{\widetilde{\delta}^{\lambda} F, G\right\} \\
& -\partial_{(i)} \partial_{k}\left[\sum_{l=0} \sigma^{A B}(-\partial)_{(j)}\left(\frac{\partial \delta_{t}^{l} \Lambda^{a}}{\partial z_{(j)}^{B}} \frac{\delta G}{\delta \lambda^{a}}\right) \frac{\delta F}{\delta z_{(i) k}^{A}}\right] \\
& -\partial_{(i)}\left[\sum_{l=0} \delta_{G}^{z}\left(\delta_{t}^{l} \Lambda^{a}\right) \frac{\delta F}{\delta \lambda^{a}{ }_{(i)}}\right]-(F \leftrightarrow G) .
\end{aligned}
$$

Using the fact that the constraints and all their derivatives are zero on the boundary along with corollary 4.2 , this becomes equation (D.2).

The cyclic identity we want to compute is between couples $\left(G_{n}, \delta_{n}^{\lambda}\right)$. Let's define

$$
\left[\left[\left(G_{1}, \delta_{1}^{\lambda}\right),\left(G_{2}, \delta_{1}^{\lambda}\right)\right],\left(G_{3}, \delta_{1}^{\lambda}\right)\right]+\text { cyclic }=\left(G_{J}, \delta_{J}^{\lambda}\right),
$$

where the bracket between couples is defined in equation (4.48). Using (D.2) and the 
Jacobi identity proven in appendix C, we obtain

$$
\begin{aligned}
G_{J}= & \widetilde{\delta}_{[1,2]}^{\lambda} G_{3}-\widetilde{\delta}_{3}^{\lambda}\left\{G_{2}, G_{1}\right\}_{\zeta}-\widetilde{\delta}_{1}^{\lambda} \widetilde{\delta}_{2}^{\lambda} G_{3}+\widetilde{\delta}_{2}^{\lambda} \widetilde{\delta}_{1}^{\lambda} G_{3} \\
& +\left\{G_{2}, \widetilde{\delta}_{3}^{\lambda} G_{1}\right\}_{\zeta}+\left\{\widetilde{\delta}_{3}^{\lambda} G_{2}, G_{1}\right\}_{\zeta}+\text { cyclic } \\
= & \sum_{l=0} \partial_{(i)}\left[\left(\delta_{t}^{l} \Lambda_{[1,2]}^{a}+\widetilde{\delta}_{2}\left(\delta_{t}^{l} \Lambda_{1}^{a}\right)-\widetilde{\delta}_{1}\left(\delta_{t}^{l} \Lambda_{2}^{a}\right)\right) \frac{\delta G_{3}}{(l)}\right]+\text { cyclic } \\
= & \sum_{l=0} \partial_{(i)}\left[\left(\left[\widetilde{\delta}_{2}, \delta_{t}^{l}\right] \Lambda_{1}^{a}-\left[\widetilde{\delta}_{1}, \delta_{t}^{l}\right] \Lambda_{2}^{a}\right) \frac{\delta G_{3}}{\delta \lambda^{(l)}}\right]+\text { cyclic }
\end{aligned}
$$

Playing with commutation relations, we obtain:

$$
\begin{aligned}
{\left[\delta_{t}, \widetilde{\delta}_{2}\right] \Lambda=} & \sigma^{A B} \partial_{(i)}\left(\left(\delta_{t} \frac{\delta G_{2}}{\delta z^{B}}-\widetilde{\delta}_{2} \frac{\delta H}{\delta z^{B}}\right) \frac{\delta \Lambda}{\delta z_{(i)}^{A}}\right) \\
= & \partial_{(i)}\left[\sigma^{A B} \frac{\delta}{\delta z^{B}}\left(\delta_{t} G_{2}-\widetilde{\delta}_{2} H-\left\{G_{2}, H\right\}\right) \frac{\delta \Lambda}{\delta z_{(i)}^{A}}\right] \\
& -\partial_{(i)}\left[(-\partial)_{(j)}\left(\frac{\partial \Lambda_{2}^{a}}{\partial z_{(j)}^{B}} \phi_{a}\right) \sigma^{B A} \frac{\delta \Lambda}{\delta z_{(i)}^{A}}\right] \\
= & -\partial_{(i)}\left[(-\partial)_{(j)}\left(\frac{\partial \Lambda_{2}^{a}}{\partial z_{(j)}^{B}} \phi_{a}\right) \sigma^{B A} \frac{\delta \Lambda}{\delta z_{(i)}^{A}}\right]
\end{aligned}
$$

where we used the fact that $\left(G_{2}, \delta_{2}^{\lambda}\right)$ generates an external symmetry. This means that, on the constraint surface, we have:

$$
G_{J} \approx 0
$$

Another useful result is that, if $\left(G_{3}, \delta_{3}^{\lambda}\right)$ is the hamiltonian $\left(H, \delta_{H}^{\lambda}\right)$, we have

$$
G_{J}=0
$$




\section{References}

[1] T. Regge and C. Teitelboim, "Role of surface integrals in the Hamiltonian formulation of general relativity," Annals of Physics 88 no. 1, (1974) 286-318.

[2] J. D. Brown and M. Henneaux, "On the Poisson brackets of differentiable generators in classical field theory," Journal of mathematical physics 27 no. 2, (1986) 489-491.

[3] R. Arnowitt, S. Deser, and C. W. Misner, "Republication of: The dynamics of general relativity," General Relativity and Gravitation 40 no. 9, (2008) 1997-2027.

[4] M. Henneaux, C. Martnez, R. Troncoso, and J. Zanelli, "Asymptotically antide Sitter spacetimes and scalar fields with a logarithmic branch," Physical Review D 70 no. 4, (2004) 044034.

[5] M. Henneaux, C. Martnez, R. Troncoso, and J. Zanelli, "Asymptotic behavior and Hamiltonian analysis of anti-de Sitter gravity coupled to scalar fields," Annals of Physics 322 no. 4, (2007) 824-848.

[6] E. Witten, "Anti-de Sitter space and holography," Adv.Theor.Math.Phys. 2 (1998) 253-291.

[7] J. A. de Azcrraga and J. M. Izquierdo, Lie groups, Lie algebras, cohomology and some applications in physics. Cambridge University Press, 1998.

[8] G. Barnich and G. Compere, "Surface charge algebra in gauge theories and thermodynamic integrability," Journal of Mathematical Physics 49 no. 4, (2008) 042901.

[9] K. Bering, "Putting an edge to the Poisson bracket," Journal of Mathematical Physics 41 no. 11, (2000) 7468-7500.

[10] M. Henneaux and C. Teitelboim, Quantization of gauge systems. Princeton university press, 1992.

[11] C. Troessaert, "Canonical Structure of Field Theories with Boundaries and Applications to Gauge Theories," arXiv preprint arXiv:1312.6427 (2013) . 